\title{
A DISTRIBUIÇÃO DIAMÉTRICA PARA BRACATINGAIS EM DIFERENTES IDADES, SÍTIOS E DENSIDADES NA REGIÃO METROPOLITANA DE CURITIBA
}

\author{
Alexandra Consuelo de Plácido e Silva Bartoszeck ${ }^{1}$ \\ Sebastião do Amaral Machado ${ }^{2}$ \\ Afonso Figueiredo Filho ${ }^{3}$ \\ Edilson Batista Oliveira ${ }^{4}$
}

\begin{abstract}
RESUMO
Esta pesquisa tem como objetivo estudar o comportamento de funções de densidade probabilística no ajuste de distribuições diamétricas para diferentes idades, sítios e densidades para povoamentos de bracatinga (Mimosa scabrella Benth) localizados nos municípios da Região Metropolitana de Curitiba, PR. Os dados utilizados são provenientes de um experimento delineado em blocos ao acaso, constituído de 5 blocos e 4 tratamentos, perfazendo um total de 20 parcelas, as quais foram remedidas 4 vezes às idades aproximadas de 4, 5, 6 e 7,5 anos de idade. A densidade inicial foi reduzida para 2000, 4000, 8000 árvores por hectare, sendo que as testemunhas não sofreram nenhum tratamento. Desses cinco blocos, um estava localizado na classe de sítio I, dois na classe II e dois na classe III. Paralelamente, foram instaladas e medidas 124 parcelas temporárias, distribuídas nos vários municípios da Região Metropolitana, representando os povoamentos de bracatinga sob o regime tradicional de cultivo e manejo, abrangendo as idades de 3 a 18 anos e as 3 classes de sítio. As seguintes funções de densidade probabilística foram ajustadas para cada combinação de idade, sítio e densidade: Normal, Lognormal, Gama, Beta, Weibull 2 parâmetros, Weibull 3 parâmetros e a $S_{B}$ de Johnson. Através do teste "D" de KolmogorovSmirnov detectou-se que a função $S_{B}$ de Johnson foi a de melhor desempenho para a maioria das combinações de dados, sendo, portanto, a primeira no ranking. Os índices de simetria e curtose indicaram que as curvas de distribuições diamétricas em bracatingais são unimodais, tornando-se cada vez mais assimétricas positivas e mais achatadas com o avanço da idade.
\end{abstract}

Palavras-chave: Mimosa scabrella, funções de densidade de probabilidade, classes diamétricas.

\section{DIAMETER DISTRIBUTION FOR STANDS OF Mimosa scabrella FOR DIFFERENT AGES, SITES AND DENSITIES}

\begin{abstract}
The objective of this research work was to study the behavior of several probability density functions in fitting the diameter distribution for different ages, sites and densities for stands of bracatinga (Mimosa scabrella Benth) located in the Curitiba metropolitan region. The utilized data came from an initial density trial, designed in random blocks, formed by 5 blocks and 4 treatments, in a total of 20 permanent sample plots with an area of $325 \mathrm{~m}^{2}$, which were remeasured 4 times at approximate ages of 4, 5, 6 and 7,5 years. The initial density was reduced for 2000, 4000, 8000 trees per hectare, remaining the control plots without any reduction of trees. From these five blocks, one was located on site class I, two on site class II and two blocks on site class III. Simultaneously they were also implanted and measured diameters and heights of every tree in 124 temporary sample plots $\left(325 \mathrm{~m}^{2}\right)$ scattered by several counties of the Curitiba metropolitan region, on stands that represent the traditional way of cultivation and management of bracatinga. These plots covered ages from 3 to 18 years and the 3 sites classes. Seven probability density functions were tested to fit the diameter distribution: Normal, Lognormal, Gama, Beta, Weibull 2 parameters, Weibull 3 parameters and the $S_{B}$ from Johnson. The combination of sites, densities and ages for the permanent and the temporary sample plots with these seven functions made it possible to accomplish 434 analysis. The Kolmogorov-Smirnov test was applied for every analysis for the choice of the best function. The Jonhson's $S_{B}$ function was the one that presented the best performance, being so, used for the construction of the diameter distribution curves. The asymmetry and Kurtosis indexes indicated that the curves of diameter distributions in bracatinga stands are unimodal, as hypothesized, becoming more and more asymmetric positively and more flattened as the stands become older.

Keywords: Mimosa scabrella, probability density functions, diameter classes
\end{abstract}

\footnotetext{
${ }^{1}$ Engenheira Florestal, M. Sc., Professora Adjunta da Uniandrade

2 Engenheiro Florestal, M.Sc., Ph.D., Professor Sênior da UFPR, Pesquisador 1A do CNPq, samac@floresta.ufpr.br

${ }^{3}$ Engenheiro Florestal , M.Sc., Dr., Prof. Adjunto da Unicentro e Prof. Sênior da UFPR, Pesquisador 1D do CNPq, afig@floresta.ufpr.br

${ }^{4}$ Engenheiro Agrônomo, Dr., Pesquisador da EMBRAPA-Florestas
}

Recebido para publicação: 13/04/2004

Aceito para publicação: 19/10/2004 


\section{INTRODUÇÃO}

Para o manejo adequado de um povoamento florestal o conhecimento da sua estrutura é fundamental. Esta consiste na distribuição de espécies e dimensões das árvores em relação a uma unidade de área, resultado dos hábitos de crescimento das espécies, das condições ambientais e práticas de manejo. Atualmente o estudo das distribuições diamétricas está amplamente difundido e aplicado em toda a Europa e Estados Unidos e, no Brasil já é bastante utilizado como subsídio para o manejo de florestas, constituindo o meio mais simples e eficaz para descrever as características de um povoamento. Os primeiros trabalhos realizados no Brasil sobre distribuições probabilísticas aplicadas a distribuições diamétricas em florestas tropicais, surgiram no limiar da década de 1980 com Barros (1980) e Finger (1982).

A distribuição diamétrica é um ótimo indicador do estoque em crescimento das florestas, sendo uma das formas mais potentes para descrever as características de um povoamento. A variável diâmetro, obtida por medição direta das árvores, é bem correlacionada com outras variáveis importantes tais como o volume, além de outras como qualidade de produção e custos de exploração. Quantificações da distribuição de diâmetro e suas relações com determinados fatores, tais como sítio, composição do povoamento, idade e densidade, são importantes para propósitos econômicos e biológicos (Bailey e Dell, 1973; Umaña, 1997).

As funções de distribuição diamétrica quando associadas às funções de afilamento, permitem a quantificação do sortimento do povoamento em pé; associando à classes de sortimentos com seus respectivos preços, permite o cálculo da renda bruta e, ainda, quando associada à uma função de produção permite estimativas da evolução dos sortimentos do povoamento, bem como da evolução de suas rendas (Machado et al., 1990).

Conhecendo a estrutura diamétrica de um povoamento, será possível avaliar melhor o comportamento da espécie a ser trabalhada, face aos vários fatores que possam vir a interferir em seu crescimento, favorecendo o manejo da mesma, considerando que todo manejo e atividades de exploração comercial provocam alterações na estrutura diamétrica. Além disso, a análise da distribuição diamétrica permite estimar o número de árvores/ha e por classe de diâmetro, bem como determinar a altura média para cada classe de diâmetro, permitindo obter informações mais detalhadas da estrutura do povoamento (Clutter et al., 1983).

Tendo em vista a importância da bracatinga, especialmente para a região metropolitana de Curitiba, e o reduzido número de estudos silvimétricos necessários para o seu manejo, esta pesquisa, partindo da hipótese que a distribuição diamétrica dos bracatingais é unimodal com diversos graus de assimetria, teve como objetivo específico ajustar e testar funções de densidade probabilística para diversas combinações de idade, sítio e densidade iniciais.

\section{MATERIAIS E MÉTODOS}

Os dados utilizados são provenientes de um experimento delineado em blocos ao acaso, constituído de 5 blocos e 4 tratamentos, perfazendo um total de 20 parcelas permanentes, as quais foram remedidas 4 vezes às idades aproximadas de 4, 5, 6 e 7,5 anos de idade. A densidade inicial foi reduzida para 2000, 4000, 8000 árvores por hectare, sendo que as testemunhas não sofreram nenhum tratamento. Desses cinco blocos, um estava localizado na classe de sítio I, dois na classe II e dois na classe III de acordo com a classificação de Machado et al. (1997), rearranjada por Tonon (1999). Paralelamente, foram instaladas e medidas 124 parcelas temporárias, distribuídas nos vários municípios da Região Metropolitana, representando os povoamentos de bracatinga sob o regime tradicional de cultivo e manejo, abrangendo as idades de 3 a 18 anos e as 3 classes de sítio.

\section{Funções de distribuições diamétricas testadas}

Foram testadas sete funções de densidade probabilística para a obtenção das distribuições de freqüências de árvores em cada classe de diâmetro para as várias combinações de idade, sítio e densidade, em 240 combinações para as parcelas permanentes e 132 para as parcelas temporárias, totalizando 372 análises (Bartoszeck, 2000). 
Para estimar os parâmetros das funções de densidade de probabilidade e posterior estimativa do número de árvores em classes diamétricas foi utilizado o pacote estatístico STATISTICA 5.1. Este pacote estima os parâmetros das funções das distribuições Normal, Lognormal, Gama e Weibull 2 parâmetros através do método da Máxima Verossimilhança e da função Beta pelo método dos Momentos. Para estimar os parâmetros das distribuições Weibull 3 parâmetros e SB de Johnson foi criada uma rotina de trabalho no programa MS EXCEL 97 utilizando o método dos Momentos.

As funções de densidade de probabilidade, correspondentes às distribuições ajustadas, são apresentadas no quadro 1 .

Quadro 1: Funções de densidade probabilística ajustadas

Table 1: Functions of density probability - fdp

\begin{tabular}{|c|c|c|}
\hline 2 & Função & Condições: \\
\hline Normal & $f(x)=\left(\frac{1}{\sigma \sqrt{2 \pi}}\right) e^{-\frac{1}{2}\left(\frac{x-\mu}{\sigma^{2}}\right)^{2}}$ & $\begin{array}{l}\sigma>0 \\
-\infty<x<+\infty \\
-\infty<\mu<+\infty\end{array}$ \\
\hline Lognormal & $f(x)=\frac{1}{x\left(\sqrt{2 \cdot \pi \cdot \sigma^{2}}\right)} \cdot e^{\left(-\frac{1}{2}\right) \frac{(\ln (x)-\mu)^{2}}{\sigma^{2}}}$ & $\begin{array}{l}x \geq a ; \sigma>0 \\
-\infty<\mu<+\infty \\
-\infty<x<+\infty\end{array}$ \\
\hline Gama & $f(x)=\frac{x^{\alpha-1} e^{\left(\frac{-x}{\beta}\right)}}{\beta^{\alpha} \cdot \Gamma(\alpha)}$ & $\begin{array}{l}x \geq a \\
-\infty<\mathrm{a}<+\infty \\
\alpha, \beta>0\end{array}$ \\
\hline Beta & $f(x)=\frac{\Gamma(\alpha+\beta)}{\Gamma(\alpha) \cdot \Gamma(\beta) \cdot(b-a)^{\alpha+\beta-1}}(x-a)^{\alpha-1}(b-x)^{\beta-1}$ & $\begin{array}{l}\mathrm{a}<x<\mathrm{b} \\
\alpha, \beta>0 \\
-\infty<\mathrm{a}<\mathrm{b}<+\infty\end{array}$ \\
\hline $\begin{array}{c}\text { Weibull } 2 \\
\text { parâmetros: }\end{array}$ & $f(x)=\frac{c}{b}\left(\frac{x}{b}\right)^{c-1} e^{-\left(\frac{x}{b}\right)^{c}}$ & $\begin{array}{l}x \geq 0 \\
c, b>0\end{array}$ \\
\hline $\begin{array}{c}\text { Weibull } 3 \\
\text { parâmetros }\end{array}$ & $f(x)=\frac{c}{b}\left(\frac{x-a}{b}\right)^{c-1} e^{-\left(\frac{x-a}{b}\right)^{c}}$ & $\begin{array}{l}x \geq \mathrm{a} \\
-\infty<\mathrm{a}<+\infty \\
\mathrm{c}, \mathrm{b}>0\end{array}$ \\
\hline$S_{B}$ de Johnson & $f(x)=\frac{\delta \lambda}{\sqrt{2 \pi}(x-\varepsilon)(\lambda+\varepsilon-x)} e^{\left[-1 / 2\left(\gamma+\delta \ln \frac{x-\varepsilon}{\lambda+\varepsilon-x}\right)^{2}\right]}$ & $\begin{array}{l}\varepsilon<\mathrm{x}<+\lambda \\
-\infty<\gamma<+\infty \\
\lambda>0, \delta>0\end{array}$ \\
\hline
\end{tabular}

legenda:

$f(x)$ - função de densidade da variável $x ; x$ - diâmetro do centro de classe; $\mu$ - média aritmética; $\sigma$ - desvio padrão; $\sigma^{2}$ é a variância da variável aleatória $x ; \pi$ - é a constante "pi" $(3,1416) ; a$ - menor valor da variável observada; $b$ - maior valor da variável observada; $\alpha, \beta, a, b, c, \varepsilon, \lambda, \delta e \gamma$-parâmetros a estimar; $\Gamma$ é a função gama caracterizada pela seguinte expressão (para qualquer $\alpha \geq 0$ ): $\Gamma(\alpha)=\int_{0}^{\infty} u^{a-1} e^{-u} \delta u$

\section{Seleção dos modelos ajustados}

Para comparar a precisão das freqüências estimadas pelos modelos de distribuição diamétrica testados com as freqüências observadas foi usado o teste $\mathrm{D}$ de Kolmogorov-Smirnov:
$D=\sup _{x}\left|F o_{(x)}-F e_{(x)}\right|$, onde:

Fo $(x)$ - freqüência observada acumulada;

$\mathrm{Fe}_{(x)}$ - freqüência estimada acumulada;

$\sup _{\mathrm{x}}$ - classe de DAP em que ocorre o mais alto valor da diferença entre $\mathrm{Fo}_{(\mathrm{x})}$ e $\mathrm{Fe}_{(\mathrm{x})}$;

$\mathrm{D}$ - ponto de maior divergência entre as freqüências 
Para a conclusão do teste, ou seja, se é ou não significativo, calcula-se: $D_{c a l}=\frac{D}{N}$ onde $\mathrm{N}$ é o número total de indivíduos da amostra. Se o $D_{\text {cal }}$ for menor que o $D_{\text {tab }}$ aceitase o ajuste. $\mathrm{D}_{\text {tab }}$ é um valor tabulado ao nível $\alpha$ de probabilidade, sendo testado para os níveis de $5 \%$ e $1 \%$ do teste bilateral.

Foram traçadas curvas das freqüências estimadas sobre o histograma das freqüências observadas por classe de diâmetro para todas as funções ajustadas. Como as análises envolveram 372 ajustes, conforme já especificado, procedeu-se com base no teste de Kolmogorov-Smirnov, a um ranking entre as sete distribuições diamétricas, para saber qual delas, na média de todas as análises, teve melhor performance.

\section{RESULTADOS E DISCUSSÕES}

\section{Caracterização dos dados das Parcelas Permanentes}

Para avaliar a estrutura diamétrica de uma floresta é necessário fazer um levantamento quantitativo dos elementos que a compõem. Algumas estatísticas descritivas foram computadas para se ter uma informação preliminar das características dessa estrutura. Estas informações são fornecidas para o total dos dados e para os dados estratificados por sítio, idade e densidade das parcelas permanentes avaliando-se a variável diâmetro à altura do peito $(\mathrm{d})$. Para o total dos dados foi encontrado uma amplitude diamétrica 0,8 a $17,2 \mathrm{~cm}$, uma média diamétrica de $6,6 \mathrm{~cm} \mathrm{e}$ um desvio padrão de 2,57 cm (tab. 1).

Com base nas amplitudes diamétricas das parcelas permanentes estratificadas por sítio, idade e densidade inicial, os dados puderam ser tabulados em classes de diâmetro com intervalos de $1 \mathrm{~cm}$, resultando em número diferente de classes para cada parcela, conforme a amplitude dos dados das mesmas.

A avaliação da concentração das árvores nas classes diamétricas inferiores ou superiores também foi um fato levantado, tomando-se como base o cálculo das freqüências relativas originadas das freqüências observadas/ha, ou seja, baseado no número de árvores/ha em percentual originados do número de árvores/ha. $\mathrm{O}$ valor médio desta freqüência relativa (\%) em relação a todas as parcelas permanentes (envolvendo todos os sítios, idades e densidades) permitiu evidenciar que a maior concentração das árvores se encontra na classe diamétrica de $7 \mathrm{a}$ $8 \mathrm{~cm}(14,2 \%)$ e que até a classe de $8 \mathrm{~cm}$ perfazem um total de $57,5 \%$ e a partir desta um percentual de $42,5 \%$. Estes resultados vêm corroborar o que foi avaliado em trabalho com bracatingais da região metropolitana de Curitiba realizado por Baggio e Carpanezzi (1997), onde relatam que em média $36,5 \%$ das árvores não ultrapassam $7 \mathrm{~cm}$ de diâmetro e maior freqüência de árvores se encontra na classe de 7,1 a $9 \mathrm{~cm}$ de diâmetro, alegando que a tendência em bracatingais é de uma concentração de árvores nas classes diamétricas inferiores, mas que a mesma pode ser influenciada pelo manejo realizado em cada bracatingal, além de outros fatores como os ambientais.

\section{Caracterização dos dados das Parcelas Temporárias}

O mesmo procedimento efetuado com as parcelas permanentes foi efetuado com as parcelas temporárias. As estatísticas descritivas das parcelas temporárias para todos os dados e para os dados separados para as combinações de sítio e idade são mostrados na Tabela 3 . Para o total dos dados (4979 árvores) foi encontrado uma amplitude diamétrica de 3,4 $\mathrm{cm}$ a $33,6 \mathrm{~cm}$, uma média diamétrica de $9,1 \mathrm{~cm}$ e um desvio padrão de $3,5 \mathrm{~cm}$. 
Tabela 1: Estatísticas descritivas das parcelas permanentes

Table 1: Descriptive statistics of the permanent sampling plots

\begin{tabular}{|c|c|c|c|c|c|c|c|c|c|c|}
\hline Sítio & Idade & $\begin{array}{c}\text { Densidade } \\
\text { Inicial }\end{array}$ & N/ha & \begin{tabular}{|c|}
$\begin{array}{c}\text { Diâmetro } \\
\text { Médio } \\
(\mathrm{cm})\end{array}$ \\
\end{tabular} & $\begin{array}{c}\text { Diâmetro } \\
\text { Mínimo (cm) }\end{array}$ & $\begin{array}{c}\text { Diâmetro } \\
\text { Máximo }(\mathrm{cm})\end{array}$ & $\begin{array}{c}\text { Variância } \\
\left(\mathrm{cm}^{2}\right)\end{array}$ & $\begin{array}{c}\text { Desvio } \\
\text { Padrão }(\mathbf{c m})\end{array}$ & Assimetria & Curtose \\
\hline \multicolumn{3}{|c|}{ Total } & 2877 & 6,6 & 0,8 & 17,2 & 6,62 & 2,57 & 0,356 & $-0,293$ \\
\hline I & 3,9 & testemunha & 5077 & 5,3 & 2,0 & 10,0 & 3,82 & 1,95 & 0,398 & $-0,832$ \\
\hline I & 3,9 & 2000 & 1477 & 9,3 & 3,0 & 13,7 & 5,51 & 2,35 & $-0,881$ & 0,816 \\
\hline I & 3,9 & 4000 & 2431 & 7,5 & 2,9 & 11,6 & 4,65 & 2,16 & $-0,107$ & $-0,626$ \\
\hline I & 3,9 & 8000 & 3200 & 6,5 & 2,0 & 11,1 & 3,86 & 1,96 & 0,061 & $-0,618$ \\
\hline I & 4,8 & testemunha & 2369 & 7,1 & 3,0 & 10,5 & 3,28 & 1,81 & 0,167 & $-0,766$ \\
\hline I & 4,8 & 2000 & 1231 & 10,0 & 2,9 & 14,7 & 5,87 & 2,42 & $-0,771$ & 0,994 \\
\hline I & 4,8 & 4000 & 2123 & 8,6 & 3,0 & 13,4 & 5,63 & 2,37 & $-0,107$ & $-0,307$ \\
\hline I & 4,8 & 8000 & 2338 & 7,7 & 3,9 & 12,0 & 3,99 & 2,00 & 0,076 & $-0,931$ \\
\hline II & 4,1 & testemunha & 5738 & 5,3 & 1,3 & 11,6 & 4,77 & 2,19 & 0,397 & $-0,541$ \\
\hline II & 4,1 & 2000 & 1862 & 8,2 & 2,0 & 13,5 & 2,96 & 1,72 & $-0,280$ & 1,565 \\
\hline II & 4,1 & 4000 & 3185 & 7,0 & 2,0 & 11,7 & 4,77 & 2,18 & $-0,194$ & $-0,679$ \\
\hline II & 4,1 & 8000 & 3877 & 6,3 & 2,0 & 13,0 & 4,89 & 2,21 & 0,201 & $-0,572$ \\
\hline II & 5 & testemunha & 3600 & 6,4 & 1,9 & 12,1 & 4,49 & 2,12 & 0,330 & $-0,495$ \\
\hline II & 5 & 2000 & 1692 & 8,5 & 3,4 & 14,9 & 3,34 & 1,83 & 0,131 & 1,460 \\
\hline II & 5 & 4000 & 2431 & 7,7 & 3,0 & 13,2 & 4,82 & 2,20 & $-0,057$ & $-0,625$ \\
\hline II & 5 & 8000 & 2738 & 7,6 & 3,0 & 13,6 & 4,53 & 2,13 & 0,269 & $-0,281$ \\
\hline II & 6,3 & testemunha & 2908 & 7,3 & 2,4 & 13,6 & 6,32 & 2,51 & 0,370 & $-0,587$ \\
\hline II & 6,3 & 2000 & 1754 & 9,4 & 4,4 & 15,9 & 3,88 & 1,97 & 0,135 & 0,227 \\
\hline II & 6,3 & 4000 & 2092 & 9,0 & 3,6 & 14,5 & 4,99 & 2,23 & 0,038 & $-0,287$ \\
\hline II & 6,3 & 8000 & 2200 & 8,5 & 3,9 & 15,0 & 5,12 & 2,26 & 0,063 & $-0,444$ \\
\hline II & 7,5 & testemunha & 954 & 8,5 & 3,7 & 14,5 & 6,18 & 2,49 & 0,257 & $-0,392$ \\
\hline II & 7,5 & 2000 & 492 & 10,6 & 6,8 & 17,2 & 5,07 & 2,25 & 0,487 & 1,009 \\
\hline II & 7,5 & 4000 & 938 & 11,3 & 7,0 & 16,7 & 5,55 & 2,36 & 0,028 & $-0,763$ \\
\hline II & 7,5 & 8000 & 954 & 9,8 & 4,9 & 16,1 & 5,00 & 2,24 & 0,155 & $-0,113$ \\
\hline III & 4,2 & testemunha & 9800 & 3,9 & 1,0 & 9,0 & 2,49 & 1,58 & 0,531 & $-0,109$ \\
\hline III & 4,2 & 2000 & 1585 & 8,0 & 3,0 & 11,3 & 2,59 & 1,61 & $-0,690$ & 1,153 \\
\hline III & 4,2 & 4000 & 2846 & 6,1 & 1,3 & 10,0 & 3,24 & 1,80 & $-0,232$ & $-0,408$ \\
\hline III & 4,2 & 8000 & 5877 & 4,8 & 0,8 & 10,5 & 2,29 & 1,51 & 0,227 & 0,262 \\
\hline III & 5,4 & testemunha & 6077 & 4,9 & 1,4 & 10,2 & 3,14 & 1,77 & 0,528 & 0,000 \\
\hline III & 5,4 & 2000 & 1477 & 8,6 & 4,9 & 12,3 & 2,74 & 1,65 & $-0,259$ & $-0,248$ \\
\hline III & 5,4 & 4000 & 2554 & 7,1 & 2,3 & 12,9 & 4,23 & 2,06 & $-0,107$ & $-0,482$ \\
\hline III & 5,4 & 8000 & 4892 & 5,3 & 1,8 & 9,5 & 2,26 & 1,50 & 0,337 & $-0,186$ \\
\hline III & 6,3 & testemunha & 4800 & 5,9 & 2,0 & 12,0 & 3,58 & 1,89 & 0,586 & 0,280 \\
\hline III & 6,3 & 2000 & 1385 & 9,5 & 5,0 & 13,8 & 3,47 & 1,86 & 0,022 & 0,080 \\
\hline III & 6,3 & 4000 & 2431 & 8,0 & 2,9 & 13,0 & 4,60 & 2,14 & $-0,098$ & $-0,518$ \\
\hline III & 6,3 & 8000 & 4154 & 6,0 & 2,8 & 10,5 & 2,27 & 1,51 & 0,399 & $-0,177$ \\
\hline III & 7,6 & testemunha & 2738 & 7,0 & 2,4 & 13,7 & 4,51 & 2,12 & 0,566 & 0,325 \\
\hline III & 7,6 & 2000 & 1062 & 10,4 & 6,3 & 14,3 & 2,70 & 1,64 & 0,070 & 0,254 \\
\hline III & 7,6 & 4000 & 1754 & 8,8 & 3,0 & 13,9 & 4,64 & 2,15 & $-0,002$ & $-0,231$ \\
\hline III & 7,6 & 8000 & 2615 & 6,8 & 2,7 & 10,8 & 2,78 & 1,67 & 0,256 & $-0,499$ \\
\hline
\end{tabular}


Tabela 2: Número de árvores/ha em porcentagem por classe diamétrica para os diferentes sítios, idades e densidades das parcelas permanentes Table 2: $\quad$ Number of trees per hectare in porcentage per diameter class for different sites, ages and densities of the permanent sampling plots

\begin{tabular}{|c|c|c|c|c|c|c|c|c|c|c|c|c|c|c|c|c|c|c|c|c|c|c|c|c|}
\hline \multirow{4}{*}{ 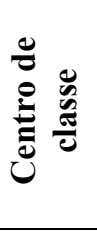 } & \multicolumn{8}{|c|}{ Sitio I } & \multicolumn{16}{|c|}{ Sítio II } \\
\hline & \multicolumn{4}{|c|}{3,9 anos } & \multicolumn{4}{|c|}{4,8 anos } & \multicolumn{4}{|c|}{4,1 anos } & \multicolumn{4}{|c|}{5,0 anos } & \multicolumn{4}{|c|}{6,3 anos } & \multicolumn{4}{|c|}{ 7,5 anos } \\
\hline & \multicolumn{24}{|c|}{ Tratamentos } \\
\hline & $\mathbf{T 1}$ & $\mathbf{T} 2$ & T3 & T4 & T1 & $\mathbf{T} 2$ & T3 & T4 & T1 & $\mathbf{T 2}$ & T3 & T4 & T1 & $\mathbf{T} 2$ & T3 & T4 & $\mathbf{T 1}$ & $\mathbf{T} 2$ & T3 & T4 & T1 & $\mathbf{T} 2$ & T3 & T4 \\
\hline 0,5 & - & - & - & - & - & - & - & - & - & - & - & - & - & - & - & - & - & - & - & - & - & - & - & - \\
\hline 1,5 & - & - & - & - & - & - & - & - & 5,4 & - & - & - & 0,4 & - & - & - & - & - & - & - & - & - & - & - \\
\hline 2,5 & 16,4 & 4,2 & 2,5 & 1,9 & - & 2,5 & - & - & 12,3 & 0,8 & 4,3 & 7,1 & 2,6 & - & - & - & 1,6 & - & - & - & - & - & - & - \\
\hline 3,5 & 24,2 & - & 2,5 & 8,7 & 5,2 & - & 4,3 & 1,3 & 18,5 & 1,7 & 9,2 & 12,7 & 11,1 & 1,8 & 4,4 & 5,1 & 5,8 & - & 0,7 & 1,4 & 4,8 & - & - & - \\
\hline 4,5 & 9,1 & 2,1 & 10,1 & 19,2 & 10,4 & 2,5 & 2,9 & 11,8 & 13,7 & 1,7 & 7,7 & 13,5 & 15,4 & 0,0 & 10,8 & 10,1 & 12,7 & 0,9 & 2,9 & 6,3 & - & - & - & 1,6 \\
\hline 5,5 & 18,2 & 4,2 & 11,4 & 9,6 & 23,4 & - & 7,2 & 11,8 & 16,6 & 6,6 & 12,6 & 15,5 & 16,2 & 6,4 & 7,6 & 15,7 & 12,7 & 1,8 & 5,9 & 10,5 & 11,3 & - & - & 3,2 \\
\hline 6,5 & 12,1 & 8,3 & 13,9 & 20,2 & 16,9 & 10,0 & 13,0 & 18,4 & 11,8 & 11,6 & 15,5 & 14,7 & 16,2 & 14,5 & 12,7 & 11,2 & 16,4 & 12,3 & 11,0 & 9,1 & 16,1 & 6,3 & 1,6 & 3,2 \\
\hline 7,5 & 10,9 & 6,3 & 20,3 & 19,2 & 14,3 & 2,5 & 18,8 & 11,8 & 10,2 & 21,5 & 16,9 & 13,5 & 16,7 & 15,5 & 19,6 & 20,2 & 16,4 & 10,5 & 18,4 & 14,7 & 19,4 & 9,4 & 8,2 & 19,4 \\
\hline 8,5 & 7,3 & 14,6 & 15,2 & 12,5 & 15,6 & 10,0 & 14,5 & 17,1 & 7,0 & 28,9 & 14,5 & 13,5 & 8,5 & 28,2 & 14,6 & 14,6 & 9,5 & 17,5 & 12,5 & 15,4 & 8,1 & 3,1 & 9,8 & 12,9 \\
\hline 9,5 & 1,8 & 20,8 & 11,4 & 6,7 & 7,8 & 17,5 & 14,5 & 15,8 & 2,1 & 17,4 & 14,0 & 5,6 & 7,3 & 16,4 & 14,6 & 12,9 & 8,5 & 16,7 & 15,4 & 13,3 & 11,3 & 18,8 & 16,4 & 9,7 \\
\hline 10,5 & - & 18,8 & 6,3 & 1,0 & 6,5 & 25,0 & 13,0 & 9,2 & 2,1 & 5,8 & 3,4 & 2,8 & 3,4 & 12,7 & 9,5 & 5,6 & 6,3 & 19,3 & 15,4 & 18,2 & 12,9 & 25,0 & 6,6 & 22,6 \\
\hline 11,5 & - & 14,6 & 6,3 & 1,0 & - & 12,5 & 2,9 & 2,6 & 0,3 & 2,5 & 1,9 & 0,8 & 1,7 & 2,7 & 5,1 & 2,2 & 7,4 & 15,8 & 10,3 & 4,9 & 6,5 & 6,3 & 14,8 & 14,5 \\
\hline 12,5 & - & 4,2 & - & - & - & 10,0 & 5,8 & - & - & 0,8 & - & 0,4 & 0,4 & 0,0 & 0,6 & 1,7 & 1,1 & 2,6 & 3,7 & 4,2 & 6,5 & 25,0 & 13,1 & 4,8 \\
\hline 13,5 & - & 2,1 & - & - & - & 5,0 & 2,9 & - & - & 0,8 & - & - & - & 0,9 & 0,6 & 0,6 & 1,6 & 1,8 & 2,2 & 1,4 & 1,6 & 3,1 & 21,3 & 6,5 \\
\hline 14,5 & - & - & - & - & - & 2,5 & 0,0 & - & - & - & - & - & - & 0,9 & - & - & - & - & 1,5 & 0,7 & 1,6 & - & 3,3 & - \\
\hline 15,5 & - & - & - & - & - & - & - & - & - & - & - & - & - & - & - & - & - & - & - & - & - & - & 3,3 & - \\
\hline 16,5 & - & - & - & - & - & - & - & - & - & - & - & - & - & - & - & - & - & - & - & - & - & - & 1,6 & 1,6 \\
\hline 17,5 & - & - & - & - & - & - & - & - & - & - & - & - & - & - & - & - & - & - & - & - & - & 3,1 & - & - \\
\hline
\end{tabular}

$\mathrm{T} 1=$ Testemunha; $\mathrm{T} 2=2000$ árvores./ha $; \mathrm{T} 3=4000$ árvores./ha; $\mathrm{T} 4=8000$ árvores./ha 
Tabela 2: Continuação

Table 2: Continuation

\begin{tabular}{|c|c|c|c|c|c|c|c|c|c|c|c|c|c|c|c|c|c|}
\hline \multirow{4}{*}{ Centro de classe } & \multicolumn{16}{|c|}{ Sitio III } & \multirow{4}{*}{$\begin{array}{l}\text { Média do número de } \\
\text { árvores/ha em } \\
\text { porcentagem nas várias } \\
\text { classes diamétricas }\end{array}$} \\
\hline & \multicolumn{4}{|c|}{4,1 anos } & \multicolumn{4}{|c|}{5,0 anos } & \multicolumn{4}{|c|}{6,3 anos } & \multicolumn{4}{|c|}{ 7,5 anos } & \\
\hline & \multicolumn{16}{|c|}{ Tratamentos } & \\
\hline & T1 & $\mathbf{T} 2$ & T3 & T4 & T1 & T2 & T3 & T4 & T1 & $\mathbf{T} 2$ & T3 & T4 & T1 & $\mathbf{T 2}$ & T3 & T4 & \\
\hline 0,5 & - & - & - & 0,8 & - & - & - & - & - & - & - & - & - & - & - & - & - \\
\hline 1,5 & 10,4 & - & 1,6 & 2,4 & 2,8 & - & - & 0,9 & - & - & - & - & - & - & - & - & 0,6 \\
\hline 2,5 & 23,4 & 1,9 & 3,8 & 10,5 & 13,2 & - & 1,8 & 4,4 & 4,2 & - & 1,9 & 0,7 & 1,1 & - & - & 0,6 & 3,1 \\
\hline 3,5 & 23,5 & 1,0 & 10,3 & 19,4 & 18,2 & - & 6,0 & 17,3 & 10,3 & - & 1,3 & 6,3 & 6,2 & - & 1,8 & 1,8 & 6,2 \\
\hline 4,5 & 20,6 & 2,9 & 16,2 & 28,3 & 25,1 & 3,1 & 10,2 & 25,2 & 21,8 & 1,1 & 5,7 & 22,2 & 7,3 & - & 0,9 & 12,4 & 9,2 \\
\hline 5,5 & 11,5 & 2,9 & 17,3 & 19,4 & 15,4 & 4,2 & 10,8 & 23,3 & 18,6 & 2,2 & 10,8 & 24,1 & 23,0 & - & 7,9 & 24,7 & 11,1 \\
\hline 6,5 & 7,7 & 16,5 & 18,4 & 13,4 & 13,2 & 11,5 & 19,3 & 17,0 & 18,9 & 5,6 & 13,3 & 23,7 & 15,2 & 1,4 & 10,5 & 20,0 & 13,1 \\
\hline 7,5 & 1,7 & 24,3 & 20,5 & 4,5 & 6,6 & 14,6 & 18,1 & 8,2 & 14,1 & 10,0 & 18,4 & 11,1 & 19,7 & 7,2 & 16,7 & 18,8 & 14,2 \\
\hline 8,5 & 1,3 & 26,2 & 8,1 & 1,0 & 3,8 & 29,2 & 13,9 & 2,8 & 6,1 & 22,2 & 14,6 & 8,5 & 10,7 & 10,1 & 21,1 & 10,6 & 12,9 \\
\hline 9,5 & - & 15,5 & 3,8 & 0,3 & 0,8 & 19,8 & 12,7 & 0,9 & 3,5 & 24,4 & 15,8 & 2,6 & 9,6 & 23,2 & 14,0 & 8,8 & 11,3 \\
\hline 10,5 & - & 7,8 & - & 0,3 & 1,0 & 11,5 & 6,6 & - & 0,6 & 15,6 & 9,5 & 0,7 & 2,8 & 30,4 & 10,5 & 2,4 & 8,8 \\
\hline 11,5 & - & 1,0 & - & - & - & 5,2 & 0,0 & - & 1,9 & 11,1 & 7,6 & - & 1,7 & 15,9 & 7,9 & - & 4,7 \\
\hline 12,5 & - & - & - & - & - & 1,0 & 0,6 & - & - & 3,3 & 1,3 & - & 2,2 & 5,8 & 7,0 & - & 2,7 \\
\hline 13,5 & - & - & - & - & - & - & - & - & - & 4,4 & - & - & 0,6 & 4,3 & 1,8 & - & 1,6 \\
\hline 14,5 & - & - & - & - & - & - & - & - & - & - & - & - & - & 1,4 & - & - & 0,3 \\
\hline 15,5 & - & - & - & - & - & - & - & - & - & - & - & - & - & - & - & - & 0,1 \\
\hline 16,5 & - & - & - & - & - & - & - & - & - & - & - & - & - & - & - & - & 0,1 \\
\hline 17,5 & - & - & - & - & - & - & - & - & - & - & - & - & - & - & - & - & 0,1 \\
\hline
\end{tabular}

$\mathrm{T} 1=$ Testemunha; $\mathrm{T} 2=2000$ árvores./ha $; \mathrm{T} 3=4000$ árvores./ha; $\mathrm{T} 4=8000$ árvores./ha 
Tabela 3: Estatísticas descritivas das parcelas temporárias

Table 3: Descriptive statistics of the temporary sampling plots

\begin{tabular}{|c|c|c|c|c|c|c|c|c|c|}
\hline \multirow{2}{*}{ Sitio } & \multirow{2}{*}{ Idade } & \multirow{2}{*}{$\begin{array}{l}\mathrm{N}^{0} \text { de árvores } \\
\text { (ha) }\end{array}$} & \multicolumn{5}{|c|}{ Diâmetro (cm) } & \multirow{2}{*}{ Assimetria } & \multirow{2}{*}{ Curtose } \\
\hline & & & Média & Mínimo & Máximo & $\begin{array}{c}\text { Variância } \\
\left(\mathrm{cm}^{2}\right)\end{array}$ & $\begin{array}{l}\text { Desvio } \\
\text { padrão }\end{array}$ & & \\
\hline \multicolumn{2}{|c|}{ Geral } & 1869 & 9,1 & 3,4 & 33,6 & 11,9 & 3,5 & 1,564 & 3,650 \\
\hline I & 3 & 3100 & 10,6 & 5,4 & 17,2 & 8,3 & 2,9 & 0,144 & $-0,336$ \\
\hline I & 4 & 3600 & 8,6 & 5,1 & 12,4 & 4,8 & 2,2 & 0,029 & $-1,162$ \\
\hline I & 5 & 2899 & 8,2 & 5,0 & 21,3 & 7,9 & 2,8 & 1,485 & 2,789 \\
\hline I & 6 & 2109 & 9,1 & 5,0 & 15,5 & 6,1 & 2,5 & 0,278 & $-0,750$ \\
\hline I & 9 & 1491 & 11,7 & 5,0 & 27,5 & 12,8 & 3,6 & 1,502 & 4,251 \\
\hline I & 10 & 700 & 14,5 & 5,3 & 25,6 & 19,8 & 4,4 & 0,087 & 0,201 \\
\hline II & 3 & 4250 & 7,2 & 5,1 & 12,4 & 2,7 & 1,6 & 1,040 & 0,720 \\
\hline II & 4 & 2525 & 8,9 & 5,1 & 16,2 & 7,8 & 2,8 & 0,653 & $-0,311$ \\
\hline II & 5 & 2179 & 7,2 & 5,0 & 14,0 & 2,9 & 1,7 & 0,920 & 0,756 \\
\hline II & 6 & 2134 & 8,6 & 5,0 & 22,0 & 5,6 & 2,4 & 0,898 & 1,589 \\
\hline II & 7 & 2010 & 9,4 & 5,0 & 19,9 & 8,4 & 2,9 & 0,779 & 0,508 \\
\hline II & $8-9$ & 1893 & 10,9 & 5,0 & 27,5 & 14,7 & 3,8 & 1,008 & 1,343 \\
\hline II & $11-12$ & 890 & 12,2 & 3,4 & 28,7 & 15,7 & 3,9 & 0,818 & 1,199 \\
\hline II & 18 & 528 & 19,0 & 6,8 & 33,6 & 24,7 & 5,0 & 0,278 & 1,380 \\
\hline III & 3 & 2700 & 8,3 & 5,4 & 12,4 & 4,7 & 2,2 & 0,312 & $-1,189$ \\
\hline III & 4 & 4300 & 8,5 & 5,1 & 15,6 & 7,2 & 2,7 & 0,662 & $-0,195$ \\
\hline III & 5 & 2478 & 6,9 & 5,0 & 13,7 & 2,5 & 1,6 & 1,080 & 1,333 \\
\hline III & 6 & 1813 & 8,2 & 5,0 & 15,0 & 4,3 & 2,1 & 0,571 & $-0,121$ \\
\hline III & 7 & 2468 & 7,3 & 5,0 & 16,7 & 3,6 & 1,9 & 1,432 & 3,238 \\
\hline III & $8-9$ & 2700 & 12,2 & 5,4 & 19,1 & 12,8 & 3,6 & 0,032 & $-0,630$ \\
\hline III & 10 & 967 & 11,3 & 3,4 & 19,5 & 20,0 & 4,5 & $-0,005$ & $-0,918$ \\
\hline III & $12-13$ & 1216 & 12,5 & 5,0 & 26,1 & 17,9 & 4,2 & 0,666 & 0,073 \\
\hline
\end{tabular}

$\begin{array}{lccr}\text { Ajuste das } & \text { Funções } & \text { de } & \text { Densidade } \\ \text { Probabilística } & \text { para } & \text { as } & \text { Parcelas } \\ \text { Permanentes } & & & \end{array}$

As sete funções de densidade probabilística, citadas anteriormente nos métodos, foram ajustadas para as 40 parcelas permanentes, resultando em 240 análises. Os resultados dos ajustes para cada uma das funções testadas e para cada combinação de sítio, idade e densidade, são apresentados na tabela 4. Como são muitas as combinações, é possível verificar a amplitude de variação dos coeficientes de cada uma das funções probabilísticas e correlacionálos com as características do povoamento.

Com base nas 240 análises, pôde-se também, montar tabelas para a comparação das distribuições. O critério estatístico para a escolha do melhor modelo de distribuição baseou-se na estatística Kolmogorov-Smirnov ao nível de 5\% e $1 \%$ de significância. A decisão da escolha ou não de determinado modelo para o ajuste das distribuições dependeu da comparação dos resultados obtidos deste teste com os valores tabelados do mesmo, como pode ser visualizado nas tabelas 5, 6 e 7, através do teste bilateral de Kolmogorov-Smirnov. Valores menores que o tabelado, indicaram a aceitação da hipótese da nulidade, o que indica que as freqüências esperadas e observadas são similares do ponto de vista estatístico, indicando que o modelo pode ser utilizado, descrevendo bem o conjunto de dados. Já, valores significativos indicam uma inadequação do uso do modelo. 
Os resultados do teste KolmogorovSmirnov (K-S) apresentaram uma amplitude de 0,0135 a 0,3360 . Na maioria das combinações estes resultados foram não significativos para o teste, $o$ que indica aderência dos dados à função proposta. Houveram casos em que a significância do teste indicou que as distribuições não são apropriadas para descrever a frequiência de diâmetros (Tabelas 5 a 7).

Ao se analisar a função $\mathrm{SB}$, procurou-se escolher a função de densidade que apresentou o menor valor de Kolmogorov-Smirnov, sendo esta função de densidade sempre associada a diferentes valores do parâmetro locação $(\varepsilon)$, que corresponde a um valor menor que o diâmetro mínimo da parcela. Avaliando este diâmetro mínimo, das 62 análises, envolvendo as parcelas permanentes e temporárias, 17 análises foram melhores quando a função estava associada ao parâmetro locação $(\varepsilon)$ a $100 \%$ do diâmetro mínimo, 13 análises com $(\varepsilon)$ à $85 \%, 9$ análises com $(\varepsilon)$ à $75 \%, 6$ análises com $(\varepsilon)$ à 95 e 65 $\%, 5$ análises com $(\varepsilon)$ à $55 \%, 4$ análises com $(\varepsilon)$ à $45 \%, 3$ análises com $(\varepsilon)$ à 5 e 25\%, 2 análises com

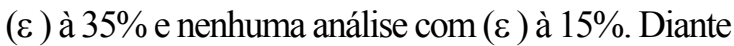
desta heterogeneidade não se pode escolher qual parâmetro foi o mais adequado, razão pela qual decidiu-se pela escolha da função com seu respectivo parâmetro que apresentasse o menor valor de Kolmogorov-Smirnov, para cada parcela ajustada.

Avaliando o sítio I, para a parcela com idade de 3,9 anos e densidade inicial testemunha, somente a função SB se ajustou bem aos dados, enquanto as outras distribuições foram inadequadas. Para o tratamento de densidade inicial 2000 árvores/ha as distribuições Lognormal, Gama, Beta e Weibull 2 parâmetros foram inadequadas, restando as distribuições Normal, Weibull 3 parâmetros e a SB para descrever os dados desta parcela. Já para a idade 4,8 anos com densidade inicial de 2000 árvores/ha a Weibull 2 parâmetros foi inadequada. Para as demais idades e densidades deste sítio todas as distribuições foram adequadas para descrever o conjunto de dados (tab. 5).

Para as parcelas com a primeira idade de medição, aos 4,1 anos, do sítio II, e tratamento de densidade inicial testemunha se ajustaram bem a Weibull 3 parâmetros, a Beta e a SB. Para a parcela com densidade de 2000 árvores/ha as distribuições Gama e Beta foram inadequadas e na densidade 4000 e 8000 árvores/ha as distribuições Lognormal e Gama não são indicadas. Para as demais idades e densidades deste sítio, todas as distribuições são adequadas para descrever o conjunto de dados (tab. $6)$.

Avaliando as parcelas do sítio III que não apresentaram adequação das distribuições, ocorreu a falta de ajuste para todas as distribuições à exceção da função SB para a primeira idade de medição, aos 4,2 anos, e testemunha. $\mathrm{Na}$ densidade 2000 árvores/ha a Weibull 2 parâmetros foi inadequada, na densidade 4000 árvores/ha a Lognormal foi inadequada e na densidade 8000 árvores/ha a Lognormal, Gama e Weibull 3 parâmetros não se ajustaram bem. Para a idade de 5,4 anos e testemunha a Normal, Lognormal e Weibull 2 não são indicadas para o uso. A Weibull 3 não se ajustou bem aos dados das parcelas com densidade 8000 árvores/ha nas idades, 5,4; 6,3 e 7,6 anos. Para as demais idades e densidades deste sítio todas as distribuições são adequadas para descrever 0 conjunto de dados (tab. 7).

Foi verificado que esta falta de ajuste, praticamente, se repetiu na primeira idade de medição para todos os sítios, independente da densidade inicial, provavelmente devido à uma distribuição diamétrica irregular nesta idade e que vai se regularizando ao longo do tempo.

Diante desta heterogeneidade, decidiu-se efetuar um ranking, o qual indicou que a função SB de Johnson ficou em primeiro lugar, seguida pelas funções Weibull 3 parâmetros, Gama, Normal, Weibull 2 parâmetros, Beta e em último a Lognormal. Logo, a função SB foi a indicada para o ajuste das distribuições diamétricas. Os valores em destaque nas Tabelas 5 a 7 indicam a função com os menores valores de Kolmogorov - Smirnov, e como pode ser observado nestas tabelas a SB aparece na maioria dos casos, como a de melhor ajuste, o que dispensaria o trabalho de realizar um ranking para a escolha da função mais adequada. Para efeito de ilustração escolheu-se um caso específico em que a linha estimativa obtida através da função SB foi traçada sobre o histograma de freqüência (fig. 1). 
Tabela 4: Estimativas dos parâmetros das funções de distribuição diamétrica para as várias combinações de sítio, idade e densidade inicial das parcelas permanentes Table 4: Estimates of parameters of the diameter distribution functions for serveral combinations of site, age and initial density of the permanent sampling plots

\begin{tabular}{|c|c|c|c|c|c|c|c|c|c|c|c|c|c|c|c|c|c|c|c|}
\hline \multirow{4}{*}{ Sitio } & \multirow{4}{*}{$\begin{array}{l}\text { Idade } \\
\text { (anos) }\end{array}$} & \multirow{4}{*}{ Densidade } & \multicolumn{17}{|c|}{ Distribuições } \\
\hline & & & \multicolumn{2}{|c|}{ Normal } & \multicolumn{2}{|c|}{ Lognormal } & \multicolumn{2}{|c|}{ Gama } & \multicolumn{2}{|c|}{ Weibull 2} & \multicolumn{3}{|c|}{ Weibull 3} & \multicolumn{2}{|c|}{ Beta } & \multicolumn{4}{|c|}{$S_{B}$} \\
\hline & & & \multicolumn{17}{|c|}{ Parâmetros } \\
\hline & & & $\mu$ & $\sigma$ & $\mu$ & $\sigma$ & $\beta$ & $\alpha$ & b & c & $\mathbf{a}$ & b & c & $\alpha$ & $\beta$ & $\varepsilon$ & $\lambda$ & $\delta$ & $\gamma$ \\
\hline $\bar{I}$ & 3,9 & testemunha & 25,27 & 1,95 & 1,59 & 0,38 & 0,733 & 7,188 & 5,921 & 2,952 & $1,1,652$ & 3,666 & 21,731 & 1,221 & 1,681 & 2,0 & 10,0 & 0,957 & $0,8,873$ \\
\hline I & 3,9 & 2000 & 9,32 & 2,35 & 2,19 & 0,32 & 0,772 & 12,078 & 11,811 & 1,556 & 2,731 & 7,040 & 2,983 & 1,397 & 1,247 & 0,8 & 13,7 & 1,208 & $-0,724$ \\
\hline I & 3,9 & 4000 & 7,49 & 2,16 & 1,97 & 0,32 & 0,690 & 10,860 & 8,284 & 3,976 & 1,826 & 4,108 & 2,767 & 2,373 & 1,935 & 2,8 & 11,6 & 1,120 & 0,496 \\
\hline I & 3,9 & 8000 & 6,48 & 1,96 & 1,82 & 0,33 & 0,648 & 10,000 & 7,189 & 3,699 & 1,714 & 5,044 & 2,450 & 1,649 & 1,701 & 1,5 & 11,1 & 1,223 & 0,294 \\
\hline I & 4,8 & testemunha & 7,05 & 1,81 & 1,92 & 0,27 & 0,478 & 14,763 & 7,747 & 4,342 & 2,780 & 4,559 & 2,405 & 1,755 & 1,734 & 3,0 & 10,5 & 1,211 & 0,658 \\
\hline I & 4,8 & 2000 & 10,01 & 2,42 & 2,27 & 0,30 & 0,744 & 13,457 & 12,513 & 1,564 & 1,736 & 8,786 & 3,785 & 3,291 & 2,099 & 1,9 & 14,7 & 1,344 & $-0,301$ \\
\hline I & 4,8 & 4000 & 8,60 & 2,37 & 2,11 & 0,31 & 0,735 & 11,708 & 9,476 & 4,122 & 2,691 & 6,290 & 2,557 & 1,641 & 1,698 & 3,0 & 13,4 & 1,202 & 0,467 \\
\hline I & 4,8 & 8000 & 7,70 & 2,00 & 2,01 & 0,27 & 0,536 & 14,349 & 8,463 & 4,359 & 2,755 & 5,279 & 2,543 & 1,992 & 1,744 & 2,5 & 12,0 & 1,310 & 0,421 \\
\hline II & 4,1 & testemunha & 5,31 & 2,19 & 1,58 & 0,45 & 0,952 & 5,577 & 5,992 & 2,640 & 0,569 & 4,865 & 2,073 & 2,048 & 3,139 & 1,0 & 11,6 & 1,067 & 0,669 \\
\hline II & 4,1 & 2000 & 8,18 & 1,72 & 2,07 & 0,25 & 0,425 & 19,234 & 8,846 & 5,230 & 1,814 & 6,761 & 3,893 & 3,533 & 3,488 & 1,1 & 13,5 & 1,767 & $-0,160$ \\
\hline II & 4,1 & 4000 & 7,04 & 2,18 & 1,89 & 0,36 & 0,791 & 8,897 & 7,817 & 3,698 & 1,672 & 5,680 & 2,475 & 1,906 & 1,945 & 0,7 & 11,7 & 1,145 & $-0,230$ \\
\hline II & 4,1 & 8000 & 6,29 & 2,21 & 1,77 & 0,38 & 0,843 & 7,461 & 7,045 & 3,136 & 1,622 & 4,829 & 2,024 & 1,635 & 2,640 & 1,3 & 13,0 & 1,225 & 0,679 \\
\hline II & 5 & testemunha & 6,44 & 2,12 & 1,80 & 0,35 & 0,725 & 8,8773 & 7,181 & 3,317 & 0,662 & 6,102 & 2,783 & 3,227 & 3,859 & 1,9 & 12,1 & 1,175 & 0,707 \\
\hline II & 5 & 2000 & 8,51 & 1,83 & 2,12 & 0,23 & 0,421 & 20,225 & 9,231 & 4,899 & 2,814 & 6,125 & 3,348 & 3,408 & 3,964 & 2,2 & 14,9 & 1,870 & 0,623 \\
\hline II & 5 & 4000 & 7,74 & 2,20 & 2,00 & 0,31 & 0,686 & 11,285 & 8,553 & 4,000 & 2,699 & 5,349 & 2,299 & 1,875 & 2,575 & 1,7 & 13,2 & 1,329 & 0,234 \\
\hline II & 5 & 8000 & 7,57 & 2,13 & 1,98 & 0,29 & 0,619 & 12,234 & 8,366 & 3,881 & 2,710 & 5,158 & 2,285 & 2,078 & 2,921 & 2,6 & 13,6 & 1,344 & 0,817 \\
\hline
\end{tabular}


A distribuição diamétrica...

Tabela 4: Continuação

Table 4: Continuation

\begin{tabular}{|c|c|c|c|c|c|c|c|c|c|c|c|c|c|c|c|c|c|c|c|}
\hline \multirow{4}{*}{ Sitio } & \multirow{4}{*}{$\begin{array}{l}\text { Idade } \\
\text { (anos) }\end{array}$} & \multirow{4}{*}{ Densidade } & \multicolumn{17}{|c|}{ Distribuições } \\
\hline & & & \multicolumn{2}{|c|}{ Normal } & \multicolumn{2}{|c|}{ Lognormal } & \multicolumn{2}{|l|}{ Gama } & \multicolumn{2}{|l|}{ Weibull 2} & \multicolumn{3}{|c|}{ Weibull 3} & \multicolumn{2}{|c|}{ Beta } & \multicolumn{4}{|c|}{$S_{B}$} \\
\hline & & & \multicolumn{17}{|c|}{ Parâmetros } \\
\hline & & & $\mu$ & $\sigma$ & $\mu$ & $\sigma$ & $\beta$ & $\alpha$ & b & c & $\mathbf{a}$ & b & c & $\alpha$ & $\beta$ & $\varepsilon$ & $\lambda$ & $\delta$ & $\gamma$ \\
\hline II & 6,3 & testemunha & 7,32 & 2,51 & 1,93 & 0,36 & 0,890 & 8,228 & 8,187 & 3,179 & 1,584 & 6,002 & 2,246 & 2,080 & 2,552 & 0,1 & 13,6 & 1,164 & 0,156 \\
\hline II & 6,3 & 2000 & 9,37 & 1,97 & 2,21 & 0,22 & 0,429 & 21,856 & 10,163 & 5,130 & 3,804 & 6,007 & 2,990 & 3,132 & 3,809 & 0,2 & 15,9 & 1,850 & $-0,605$ \\
\hline II & 6,3 & 4000 & 8,96 & 2,23 & 2,16 & 0,27 & 0,595 & 15,056 & 9,823 & 4,470 & 2,732 & 6,678 & 2,918 & 2,954 & 2,982 & 3,1 & 14,5 & 1,419 & 0,598 \\
\hline II & 6,3 & 8000 & 8,51 & 2,26 & 2,10 & 0,28 & 0,640 & 13,285 & 9,361 & 4,193 & 2,709 & 6,190 & 2,628 & 2,622 & 3,109 & 1,4 & 15,0 & 1,503 & 0,160 \\
\hline II & 7,5 & testemunha & 8,46 & 2,49 & 2,09 & 0,31 & 0,756 & 11,188 & 9,366 & 3,748 & 2,655 & 6,140 & 2,360 & 2,125 & 2,521 & 2,8 & 14,5 & 1,227 & 0,628 \\
\hline II & 7,5 & 2000 & 10,65 & 2,25 & 2,34 & 0,21 & 0,460 & 23,148 & 11,549 & 4,935 & 5,810 & 5,212 & 2,232 & 2,069 & 3,215 & 5,8 & 17,2 & 1,450 & 1,498 \\
\hline II & 7,5 & 4000 & 11,27 & 2,36 & 2,40 & 0,22 & 0,502 & 22,461 & 12,224 & 5,407 & 6,773 & 4,808 & 1,907 & 2,154 & 2,312 & 3,9 & 16,7 & 1,611 & 0,393 \\
\hline II & 7,5 & 8000 & 9,80 & 2,24 & 2,25 & 0,24 & 0,524 & 18,704 & 10,676 & 4,800 & 3,766 & 6,480 & 2,842 & 3,173 & 3,931 & 2,2 & 16,1 & 1,655 & 0,207 \\
\hline III & 4,2 & testemunha & 3,91 & 1,58 & 1,28 & 0,43 & 0,663 & 5,894 & 4,407 & 2,665 & 0,694 & 3,281 & 1,921 & 1,582 & 2,736 & 0,9 & 9,0 & 1,124 & 0,888 \\
\hline III & 4,2 & 2000 & 7,99 & 1,61 & 2,05 & 0,24 & 0,391 & 20,419 & 10,242 & 1,484 & 2,848 & 5,534 & 3,452 & 4,686 & 3,202 & 2,6 & 11,3 & 1,610 & 0,132 \\
\hline III & 4,2 & 4000 & 6,07 & 1,80 & 1,75 & 0,35 & 0,642 & 9,465 & 6,718 & 3,859 & 0,741 & 5,667 & 3,092 & 2,483 & 1,899 & 0,3 & 10,0 & 1,181 & $-0,419$ \\
\hline III & 4,2 & 8000 & 4,81 & 1,51 & 1,51 & 0,36 & 0,532 & 9,033 & 5,342 & 3,446 & $-0,23$ & 5,339 & 3,533 & 4,876 & 6,394 & 0,8 & 10,5 & 1,504 & 0,803 \\
\hline III & 5,4 & testemunha & 4,95 & 1,77 & 1,53 & 0,37 & 0,647 & 7,645 & 5,546 & 3,000 & 0,693 & 4,454 & 2,374 & 2,638 & 3,983 & 1,4 & 10,2 & 1,150 & 0,855 \\
\hline III & 5,4 & 2000 & 8,63 & 1,65 & 2,14 & 0,21 & 0,340 & 25,348 & 9,297 & 6,029 & 3,854 & 5,164 & 3,082 & 3,225 & 3,084 & 2,7 & 12,3 & 1,722 & 0,132 \\
\hline III & 5,4 & 4000 & 7,14 & 2,06 & 1,92 & 0,32 & 0,670 & 10,650 & 7,889 & 3,939 & 1,713 & 5,771 & 2,703 & 2,632 & 3,020 & 1,0 & 12,9 & 1,404 & 0,171 \\
\hline III & 5,4 & 8000 & 5,29 & 1,50 & 1,62 & 0,30 & 0,442 & 11,973 & 5,851 & 3,804 & 0,792 & 4,795 & 3,133 & 3,734 & 4,053 & 1,5 & 9,5 & 1,360 & 0,651 \\
\hline III & 6,3 & testemunha & 5,94 & 1,89 & 1,73 & 0,33 & 0,602 & 9,865 & 6,616 & 3,335 & 1,707 & 4,451 & 2,205 & 1,912 & 2,857 & 1,7 & 12,0 & 1,307 & 0,901 \\
\hline III & 6,3 & 2000 & 9,48 & 1,86 & 2,23 & 0,21 & 0,381 & 24,893 & 10,242 & 5,603 & 4,831 & 5,038 & 2,608 & 3,050 & 2,471 & 3,8 & 13,8 & 1,669 & 0,620 \\
\hline III & 6,3 & 4000 & 7,98 & 2,14 & 2,04 & 0,30 & 0,641 & 12,450 & 8,780 & 4,229 & 1,721 & 6,678 & 3,057 & 2,944 & 2,516 & 1,3 & 13,0 & 1,349 & $-0,081$ \\
\hline III & 6,3 & 8000 & 6,04 & 1,51 & 1,77 & 0,25 & 0,376 & 16,067 & 6,631 & 4,287 & 1,817 & 4,532 & 2,927 & 3,522 & 4,268 & 2,7 & 10,5 & 1,400 & 1,167 \\
\hline III & 7,6 & testemunha & 7,02 & 2,12 & 1,90 & 0,31 & 0,642 & 10,938 & 7,790 & 3,506 & 1,689 & 5,650 & 2,542 & 2,699 & 3,674 & 0,1 & 13,7 & 1,458 & $-0,023$ \\
\hline III & 7,6 & 2000 & 10,37 & 1,64 & 2,33 & 0,16 & 0,264 & 39,263 & 11,063 & 6,804 & 5,893 & 4,879 & 2,922 & 2,882 & 3,070 & 6,0 & 14,3 & 1,756 & 1,545 \\
\hline III & 7,6 & 4000 & 8,82 & 2,15 & 2,14 & 0,27 & 0,570 & 15,478 & 9,651 & 4,576 & 2,748 & 6,509 & 2,959 & 2,183 & 2,005 & 3,0 & 13,9 & 1,419 & 0,523 \\
\hline III & 7,6 & 8000 & 6,79 & 1,67 & 1,88 & 0,25 & 0,419 & 16,215 & 7,440 & 4,444 & 1,801 & 5,343 & 3,160 & 3,189 & 2,751 & 2,7 & 10,8 & 1,379 & 0,772 \\
\hline
\end{tabular}


Tabela 5: Estatística Kolmogorov-Smirnov para as distribuições probabilísticas testadas para as parcelas permanentes nas idades 3,9 e 4,8 anos, nos 4 tratamentos de densidade inicial e no sítio I

Table 5: Kolmogorov-Smirnov statistic for the tested probabilistic distributions for the permanent sampling plots at ages 3.9 and 4.8 years for the initial density treatments Site I

\begin{tabular}{|c|c|c|c|c|c|c|c|c|}
\hline \multirow{3}{*}{ Função } & \multicolumn{4}{|c|}{ Idade 3,9} & \multicolumn{4}{|c|}{ Idade 4,8} \\
\hline & \multicolumn{4}{|c|}{ Tratamentos } & \multicolumn{4}{|c|}{ Tratamentos } \\
\hline & Testemunha & 2000 & 4000 & 8000 & Testemunha & 2000 & 4000 & 8000 \\
\hline Normal & $0,148^{* *}$ & $0,154^{\mathrm{ns}}$ & $0,054^{\mathrm{ns}}$ & $0,079^{\mathrm{ns}}$ & $0,115^{\mathrm{ns}}$ & $0,117^{\mathrm{ns}}$ & $0,064^{\mathrm{ns}}$ & $0,081^{\mathrm{ns}}$ \\
\hline Lognormal & $0,108^{*}$ & $0,217^{*}$ & $0,092^{\mathrm{ns}}$ & $0,095^{\mathrm{ns}}$ & $0,089^{\mathrm{ns}}$ & $0,171^{\mathrm{ns}}$ & $0,105^{\mathrm{ns}}$ & $0,094^{\mathrm{ns}}$ \\
\hline Gama & $0,125^{*}$ & $0,199^{*}$ & $0,082^{\mathrm{ns}}$ & $0,082^{\mathrm{ns}}$ & $0,090^{\mathrm{ns}}$ & $0,145^{\mathrm{ns}}$ & $0,093^{\mathrm{ns}}$ & $0,085^{\mathrm{ns}}$ \\
\hline Weibull 2 & $0,136^{* *}$ & $0,296^{* *}$ & $0,045^{\mathrm{ns}}$ & $0,076^{\mathrm{ns}}$ & $0,120^{\mathrm{ns}}$ & $0,291^{* *}$ & $0,072^{\mathrm{ns}}$ & $0,084^{\mathrm{ns}}$ \\
\hline Weibull 3 & $0,078^{* *}$ & $0,111^{\mathrm{ns}}$ & $0,077^{\mathrm{n}}$ & $0,095^{\mathrm{ns}}$ & $0,048^{\mathrm{ns}}$ & $0,110^{\mathrm{ns}}$ & $0,041^{\mathrm{ns}}$ & $0,073^{\mathrm{ns}}$ \\
\hline Beta & $0,103^{* *}$ & $0,219^{*}$ & $0,049^{\mathrm{ns}}$ & $0,074^{\mathrm{ns}}$ & $0,095^{\mathrm{ns}}$ & $0,111^{\mathrm{ns}}$ & $0,145^{\mathrm{ns}}$ & $0,073^{\mathrm{ns}}$ \\
\hline $\mathbf{S}_{\mathrm{B}}$ & $0,066^{\mathrm{ns}}$ & $0,072^{\mathrm{ns}}$ & $0,049^{\mathrm{ns}}$ & $0,042^{\mathrm{ns}}$ & $0,061^{\mathrm{ns}}$ & $0,069^{\mathrm{ns}}$ & $0,057^{\mathrm{ns}}$ & $0,046^{\mathrm{ns}}$ \\
\hline
\end{tabular}

Legenda: ns - não significativo; * - significativo à $5 \%$; ** - significativo à $1 \%$. Testemunha - tratamento de densidade inicial testemunha

Tabela 6: Estatística Kolmogorov-Smirnov para as distribuições probabilísticas testadas para as parcelas permanentes nas idades 4,1 a 7,5 anos, nos 4 tratamentos de densidade inicial e no sítio II

Table 6: Kolmogorov-Smirnov statistic for the tested probabilistic distributions for the permanent sampling plots at ages 4.1 to 7.5 years for the initial density treatments Site II

\begin{tabular}{|c|c|c|c|c|c|c|c|c|c|c|c|c|c|c|c|c|}
\hline \multirow{3}{*}{ Função } & \multicolumn{4}{|c|}{ Idade $\mathbf{4 , 1}$} & \multicolumn{4}{|c|}{ Idade $\mathbf{5 , 0}$} & \multicolumn{4}{|c|}{ Idade 6,3} & \multicolumn{4}{|c|}{ Idade 7,5} \\
\hline & \multicolumn{4}{|c|}{ Tratamentos } & \multicolumn{4}{|c|}{ Tratamentos } & \multicolumn{4}{|c|}{ Tratamentos } & \multicolumn{4}{|c|}{ Tratamentos } \\
\hline & Test. & 2000 & 4000 & 8000 & Test. & 2000 & 4000 & 8000 & Test. & 2000 & 4000 & 8000 & Test. & 2000 & 4000 & 8000 \\
\hline Normal & $0,088^{* *}$ & $0,068^{\mathrm{ns}}$ & $0,077^{\mathrm{ns}}$ & $0,099^{\mathrm{ns}}$ & $0,063^{\text {ns }}$ & $0,081^{\mathrm{ns}}$ & $0,060^{\mathrm{ns}}$ & $0,079^{\mathrm{ns}}$ & $0,074^{\mathrm{ns}}$ & $0,047^{\mathrm{ns}}$ & $0,057^{\mathrm{ns}}$ & $0,060^{\mathrm{ns}}$ & $6,638^{\mathrm{ns}}$ & $0,085^{\mathrm{ns}}$ & $0,099^{\mathrm{ns}}$ & $0,117^{\mathrm{ns}}$ \\
\hline Lognormal & $0,087^{* *}$ & $0,119^{\mathrm{ns}}$ & $0,141^{* *}$ & $0,114^{* *}$ & $0,063^{\text {ns }}$ & $0,128^{\mathrm{ns}}$ & $0,107^{\mathrm{ns}}$ & $0,102^{\mathrm{ns}}$ & $0,057^{\mathrm{ns}}$ & $0,082^{\mathrm{ns}}$ & $0,065^{\mathrm{ns}}$ & $0,090^{\mathrm{ns}}$ & $0,080^{\mathrm{ns}}$ & $0,090^{\mathrm{ns}}$ & $0,113^{\mathrm{ns}}$ & $0,110^{\mathrm{ns}}$ \\
\hline Gama & $0,070^{*}$ & $0,102^{*}$ & $0,122^{* *}$ & $0,099^{* *}$ & $0,052^{\mathrm{ns}}$ & $0,111^{\mathrm{ns}}$ & $0,086^{\mathrm{ns}}$ & $0,083^{\mathrm{ns}}$ & $0,058^{\mathrm{ns}}$ & $0,069^{\mathrm{ns}}$ & $0,056^{\mathrm{ns}}$ & $0,071^{\mathrm{ns}}$ & $0,064^{\mathrm{ns}}$ & $0,083^{\mathrm{ns}}$ & $0,109^{\mathrm{ns}}$ & $0,103^{\mathrm{ns}}$ \\
\hline Weibull 2 & $0,071^{*}$ & $0,077^{\mathrm{ns}}$ & $0,070^{\mathrm{ns}}$ & $0,061^{\mathrm{ns}}$ & $0,053^{\mathrm{ns}}$ & $0,082^{\mathrm{ns}}$ & $0,056^{\mathrm{ns}}$ & $0,068^{\mathrm{ns}}$ & $0,062^{\mathrm{ns}}$ & $0,056^{\mathrm{ns}}$ & $0,060^{\mathrm{ns}}$ & $0,062^{\mathrm{ns}}$ & $0,093^{\mathrm{ns}}$ & $0,104^{\mathrm{ns}}$ & $0,083^{\mathrm{ns}}$ & $0,109^{\mathrm{ns}}$ \\
\hline Weibull 3 & $0,062^{\mathrm{ns}}$ & $0,078^{\mathrm{ns}}$ & $0,081^{\mathrm{ns}}$ & $0,079^{\mathrm{ns}}$ & $0,051^{\mathrm{ns}}$ & $0,054^{\mathrm{ns}}$ & $0,100^{\mathrm{ns}}$ & $0,060^{\mathrm{ns}}$ & $0,067^{\mathrm{ns}}$ & $0,070^{\mathrm{ns}}$ & $0,053^{\mathrm{ns}}$ & $0,079^{\mathrm{ns}}$ & $0,073^{\mathrm{ns}}$ & $0,097^{\mathrm{ns}}$ & $0,117^{\mathrm{ns}}$ & $0,092^{\mathrm{ns}}$ \\
\hline Beta & $0,056^{\mathrm{ns}}$ & $0,129^{*}$ & $0,093^{\mathrm{ns}}$ & $0,075^{\mathrm{ns}}$ & $0,047^{\mathrm{ns}}$ & $0,110^{\mathrm{ns}}$ & $0,107^{\mathrm{ns}}$ & $0,083^{\mathrm{ns}}$ & $0,066^{\mathrm{ns}}$ & $0,076^{\mathrm{ns}}$ & $0,047^{\mathrm{ns}}$ & $0,084^{\mathrm{ns}}$ & $0,077^{\mathrm{ns}}$ & $0,119^{\mathrm{ns}}$ & $0,087^{\mathrm{ns}}$ & $0,092^{\mathrm{ns}}$ \\
\hline $\mathbf{S}_{\mathbf{B}}$ & $0,033^{\mathrm{ns}}$ & $0,048^{\mathrm{ns}}$ & $0,043^{\mathrm{ns}}$ & $0,031^{\mathrm{ns}}$ & $0,019^{\mathrm{ns}}$ & $0,049^{\mathrm{ns}}$ & $0,041^{\mathrm{ns}}$ & $0,046^{\mathrm{ns}}$ & $0,086^{\mathrm{ns}}$ & $0,038^{\mathrm{ns}}$ & 0,027 & 0,037 & 0,023 & $0,089^{\mathrm{ns}}$ & $0,055^{\mathrm{ns}}$ & $0,048^{\mathrm{ns}}$ \\
\hline
\end{tabular}

Legenda: ns - não significativo; * - significativo à $5 \%$; ** - significativo à $1 \%$. Test. - tratamento de densidade inicial testemunha 
Tabela 7: Estatística Kolmogorov-Smirnov para as distribuições probabilísticas testadas para as parcelas permanentes nas idades 4,2 a 7,6 anos, nos 4 tratamentos de densidade inicial e no sítio III

Table 7: $\quad$ Kolmogorov-Smirnov statistic for the tested probabilistic distributions for the permanent sampling plots at ages 4.2 to 7.6 years for the initial density treatments -

\begin{tabular}{|c|c|c|c|c|c|c|c|c|c|c|c|c|c|c|c|c|}
\hline \multirow{3}{*}{ Função } & \multicolumn{4}{|c|}{ Idade 4,2 } & \multicolumn{4}{|c|}{ Idade $\mathbf{5 , 4}$} & \multicolumn{4}{|c|}{ Idade 6,3} & \multicolumn{4}{|c|}{ Idade 7,6 } \\
\hline & \multicolumn{4}{|c|}{ Tratamentos } & \multicolumn{4}{|c|}{ Tratamentos } & \multicolumn{4}{|c|}{ Tratamentos } & \multicolumn{4}{|c|}{ Tratamentos } \\
\hline & Test. & 2000 & 4000 & 8000 & Test. & 2000 & 4000 & 8000 & Test. & 2000 & 4000 & 8000 & Test. & 2000 & 4000 & 8000 \\
\hline & "0,096 & 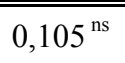 & $0,0,079^{\text {ns }}$ & 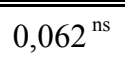 & "0,090** & 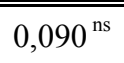 & 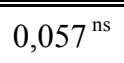 & $0,072^{\mathrm{ns}}$ & 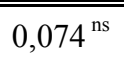 & 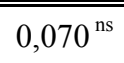 & $0,043^{\mathrm{ns}}$ & 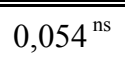 & 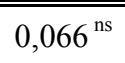 & 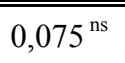 & 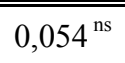 & $0,085^{\mathrm{ns}}$ \\
\hline Lognormal & $0,101^{* *}$ & $0,146^{\mathrm{ns}}$ & $0,104^{* *}$ & $0,095^{*}$ & $0,088^{* *}$ & $0,127^{\mathrm{ns}}$ & $0,096^{\text {ns }}$ & $0,073^{\mathrm{ns}}$ & $0,046^{\mathrm{ns}}$ & $0,101^{\mathrm{ns}}$ & $0,086^{\mathrm{ns}}$ & $0,041^{\mathrm{ns}}$ & $0,058^{\mathrm{ns}}$ & $0,081^{\mathrm{ns}}$ & $0,072^{\text {ns }}$ & $0,049^{\mathrm{ns}}$ \\
\hline na & $0,077^{* *}$ & $0,134^{\mathrm{ns}}$ & $0,100^{\mathrm{ns}}$ & $0,071^{*}$ & $0,067^{\mathrm{ns}}$ & $0,116^{\mathrm{ns}}$ & $0,076^{\text {ns }}$ & $0,058^{\mathrm{ns}}$ & $0,036^{\mathrm{ns}}$ & $0,088^{\mathrm{ns}}$ & $0,069^{\mathrm{ns}}$ & $0,036^{\mathrm{ns}}$ & $0,040^{\mathrm{ns}}$ & $0,070^{\mathrm{ns}}$ & $0,057^{\mathrm{ns}}$ & $0,056^{\mathrm{ns}}$ \\
\hline Weibull 2 & $0,075^{* *}$ & $0,336^{* *}$ & $0,071^{\text {ns }}$ & $0,064^{\mathrm{ns}}$ & $0,075^{*}$ & $0,089^{\text {ns }}$ & $0,053^{\text {ns }}$ & $0,075^{\text {ns }}$ & $0,064^{\mathrm{ns}}$ & $0,095^{\mathrm{ns}}$ & $0,039^{\mathrm{ns}}$ & $0,061^{\text {ns }}$ & $0,065^{\text {ns }}$ & $0,107^{\mathrm{ns}}$ & $0,071^{\mathrm{ns}}$ & $0,088^{\mathrm{ns}}$ \\
\hline Weibull 3 & $0,065^{* *}$ & $0,057^{\mathrm{ns}}$ & $0,068^{\mathrm{ns}}$ & $0,026^{* *}$ & $0,047^{\mathrm{ns}}$ & $0,065^{\mathrm{ns}}$ & $0,072^{\mathrm{ns}}$ & $0,031^{* *}$ & $0,063^{\mathrm{ns}}$ & $0,069^{\mathrm{ns}}$ & $0,070^{\mathrm{ns}}$ & $0,041^{* *}$ & $0,081^{\mathrm{ns}}$ & $0,046^{\mathrm{ns}}$ & $0,037^{\mathrm{ns}}$ & $0,035^{* *}$ \\
\hline Beta & $0,080^{* *}$ & $0,096^{\mathrm{ns}}$ & $0,075^{\mathrm{ns}}$ & $0,057^{\text {ns }}$ & $0,068^{\mathrm{ns}}$ & $0,091^{\mathrm{ns}}$ & $0,071^{\text {ns }}$ & $0,071^{\mathrm{ns}}$ & $0,070^{\text {ns }}$ & $0,115^{\mathrm{ns}}$ & $0,044^{\mathrm{ns}}$ & $0,053^{\text {ns }}$ & $0,073^{\text {ns }}$ & $0,091^{\mathrm{ns}}$ & $0,090^{\mathrm{ns}}$ & $0,084^{\mathrm{ns}}$ \\
\hline$S_{\mathbf{B}}$ & $0,014^{\mathrm{ns}}$ & $0,035^{\mathrm{ns}}$ & $0,039^{\text {ns }}$ & $0,034^{\mathrm{ns}}$ & $0,030^{\mathrm{ns}}$ & $0,037^{\text {ns }}$ & $0,023^{\text {ns }}$ & $0,020^{\mathrm{ns}}$ & $0,027^{\text {ns }}$ & $0,04^{\mathrm{ns}}$ & $0,016^{\mathrm{ns}}$ & $0,015^{\text {ns }}$ & $0,037^{\text {ns }}$ & $0,044^{\mathrm{ns}}$ & $0,034^{\mathrm{ns}}$ & $0,036^{\mathrm{ns}}$ \\
\hline
\end{tabular}

Legenda: ns - não significativo; * - significativo à 5\%; ** - significativo à 1\%. Test. - tratamento de densidade inicial testemunha 


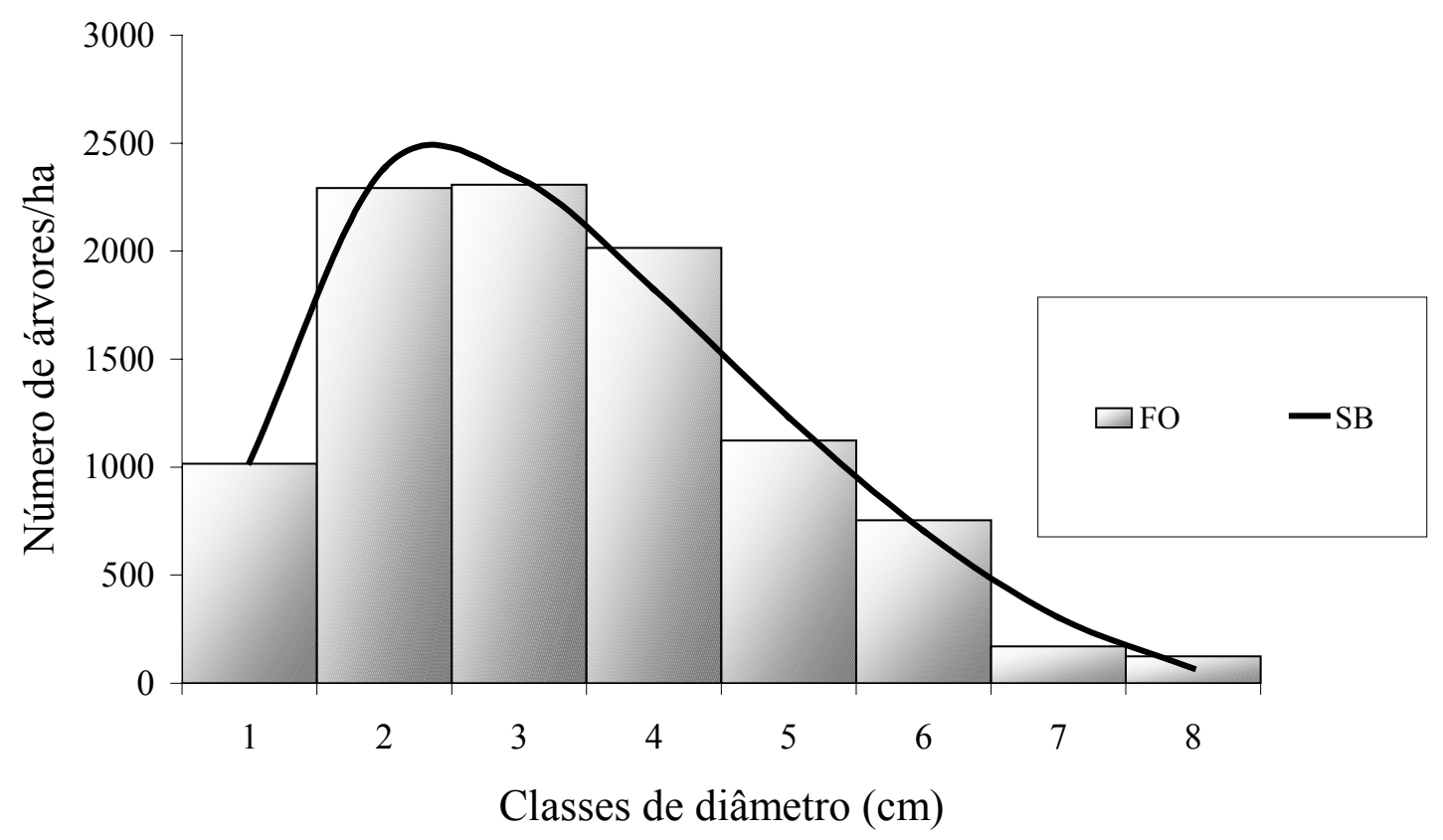

Figura 1: Curvas da distribuição diamétrica estimadas pela função $\mathrm{S}_{\mathrm{B}}$ para a parcela do sítio I, idade 3,9 anos e testemunha

Figure 1: Diameter distribution curves estimated by the $S_{B}$ function for the Site I plot, age 3.9 years and control plot

Legenda: FO - freqüência observada, SB - Curva estimada pela função SB

$\begin{array}{lccr}\text { Ajuste das } & \text { Funções } & \text { de } & \text { Densidade } \\ \text { Probabilística } & \text { para } & \text { as } & \text { Parcelas } \\ \text { Temporárias } & & & \end{array}$

Para cada combinação de sítio e idade foram ajustadas as sete funções de densidade probabilística citadas anteriormente resultando em 132 análises. Estas análises forneceram resultados do teste Kolmogorov-Smirnov (KS) de 0,0219 a 0,1610 .

Para as parcelas do sítio I, nas idades 3, 4, 9 e 10-11 anos todas as funções tiveram bons desempenhos, visto que não apresentaram significância nos valores dos testes estatísticos. Para a idade 5 somente a SB e a Weibull 3 parâmetros foram adequadas e as outras funções apresentaram significância nos valores dos testes estatísticos, indicando inadequabilidade de uso (tab. 9). A mesma falta de ajuste ocorreu para as parcelas do sítio II nas idades 5 e 6 para todas as distribuições. $\mathrm{Na}$ idade 7, a falta de ajuste apareceu para a distribuições Weibull 2 parâmetros e Beta e, na idade 11-12 para a Normal e Weibull 2 parâmetros (tab. 10).

Avaliando as parcelas do sítio III a falta de ajuste ocorreu, nas idades 5 e $7 \mathrm{com}$ todas as distribuições à exceção da função SB. $\mathrm{Na}$ idade 12-13 somente a Normal não se adequou. Para as outras idades todas as distribuições podem ser utilizadas (tab. 10).

Ao se fazer um ranking das distribuições em função do K-S pôde-se concluir que em primeiro lugar apareceu a função SB, seguida pelas funções Lognormal, Gamma, Weibull 3 parâmetros, Beta, Normal e por último a Weibull 2 parâmetros.

Os valores do K-S para todas as análises efetuadas para as parcelas temporárias se encontram na Tabelas 9 e 10 . Os valores destacados indicam a função com o menor valor. Como pode ser observado nessas tabelas, a SB aparece na maioria dos casos como a de melhor ajuste igualmente como ocorreu com as parcelas permanentes. 
A distribuição diamétrica...

Tabela 8: Estimativas dos parâmetros das funções de distribuição diamétrica para as várias combinações de sítio, idade e densidade inicial das parcelas temporárias

Table 8: Estimates of the diameter distribution functions parameters for the several combinations of site, age and initial density of the temporary sampling plots

\begin{tabular}{|c|c|c|c|c|c|c|c|c|c|c|c|c|c|c|c|c|c|c|}
\hline \multirow{4}{*}{ Sitio } & \multirow{4}{*}{$\begin{array}{l}\text { Idade } \\
\text { (anos) }\end{array}$} & \multicolumn{17}{|c|}{ Distribuições } \\
\hline & & \multicolumn{2}{|c|}{ Normal } & \multicolumn{2}{|c|}{ Lognormal } & \multicolumn{2}{|c|}{ Gama } & \multicolumn{2}{|c|}{ Weibull 2} & \multicolumn{3}{|c|}{ Weibull 3} & \multicolumn{2}{|c|}{ Beta } & \multicolumn{4}{|c|}{$\mathbf{S}_{\mathbf{B}}$} \\
\hline & & \multicolumn{17}{|c|}{ Parâmetros } \\
\hline & & $\mu$ & $\Sigma$ & $\mu$ & $\sigma$ & $\beta$ & $\alpha$ & $\mathbf{b}$ & c & $\mathbf{A}$ & b & c & $\alpha$ & $\beta$ & $\varepsilon$ & $\lambda$ & $\delta$ & $\gamma$ \\
\hline I & 3 & & & & 29 & 10 & & 11,631 & 4,101 & 4,653 & 6,233 & 2,064 & 1,577 & 2,141 & 3,5 & 17,2 & 1,277 & 0,53 \\
\hline I & 4 & 63 & 2,19 & 2,12 & 26 & 564 & ,309 & 470 & 553 & 4,752 & 060 &, 743 & 1,022 & 1,312 & 3,8 & 12,4 & 1,181 & 0,63 \\
\hline I & 5 & 23 & 2,81 & 206 & 031 & 802 & 10,269 & 9199 & 958 & 4,536 & 401 & 156 & 0,751 & 3,189 & 4,8 & 21,3 & 1,015 & 1,98 \\
\hline I & 6 & & 2,47 & 2,1 & & 672 & 613 & 10,085 & 4,070 & 4,677 & 4,650 & 1,738 & 1,188 & 2,032 & 3,8 & 15,5 & 1,281 & 0,92 \\
\hline I & 9 & 73 & 3,5 & 2,42 & 20 & 953 & 12,307 & 13,013 & 3,228 & 4,473 & 7,590 & 1,973 & 1,794 & 4,136 & 5,0 & 27,5 & 1,338 & , \\
\hline I & 10 & 14,50 & 4,45 & 2,62 & 0,35 & 1,522 & 9,528 & 16,079 & 3,611 & 4,359 & 10,695 & 2,294 & 1,606 & 1,968 & 2,9 & 25,6 & 1,255 & 0,27 \\
\hline II & 3 & 16 & 1,65 & 1,94 & & 340 & 21,075 & 7,824 & 4,347 & 4,817 & 2,349 &, 332 & 1,099 & 2,868 & 4,3 & 12,4 & 1,249 & 1,74 \\
\hline II & 4 & & 2,79 & 2,17 & & 324 & & + & 3 & 4 & + &, 443 & 1,011 & 2,057 & 4,3 & 16,2 & 51 & 1,1 \\
\hline II & 5 & 7,17 & 1,71 & 1,94 & 0,23 & 0,374 & 19,190 & 7,853 & 4,225 & 4,800 & 2,348 & 1,286 & 0,708 & 2,266 & 4,3 & 14,0 & 1,297 & 1,95 \\
\hline II & 6 & & 2,37 & 2,1 & & 0,611 & 14,154 & प,ग00 & 3,696 & 4,682 & 4,059 & 1,574 & 1,141 & 4,232 & 3,8 & 22,0 & 545 & 2,10 \\
\hline II & 7 & & 2,89 & 2,1 & & 0,847 & 11,057 & 10,409 & 3,383 & 4,564 & 4,847 & 1,541 & 1,077 & 2,642 & 8 & 19,9 & 1,282 & 1,3 \\
\hline II & 8 & 1093 & 3,84 & 23 & 0,34 & 1,238 & 8,824 & 12,231 & 2,963 & 4,349 & 6,601 & 1,582 & 1,410 & 3,997 & 3,8 & 27,5 & 1,283 & 1,52 \\
\hline II & 11 & & 3, & & & & & 13,610 & 3,207 & 2,375 & 10,385 & 2 & 2,951 & 5,256 & 3,4 & 28,7 & 1,426 & 1, \\
\hline II & 10 & 18,97 & 4,97 & 2,11 & & 1,402 & 13,531 & 20,822 & 4,060 & 5,394 & 14,486 & 2,869 & 2,430 & 2,753 & 1,7 & 33,6 & 1,541 & $-0,0$ \\
\hline W & 3 & & 2,18 & 208 & & 0,549 & & & & & & & & 1,631 & 4,6 & 12,4 & 1,049 & 1,0 \\
\hline III & 4 & & 2,69 & 2,0 & & & & 9,446 & 3,375 & 4 , & 3,776 & 16 & 0,812 & 1,734 & 7,9 & 15,6 & 0,935 & 1,3 \\
\hline III & 5 & 6,91 & 1,59 & 1,91 & & 0,332 & 20,837 & 7,555 & 4,272 & 4,820 & 2,040 & 1,210 & 0,681 & 2,604 & 4,3 & 13,7 & 1,301 & 2,08 \\
\hline WU & 6 & & 2,0 & 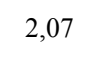 & & 0,501 & 16,360 & 8,995 & 4, & 4,747 & 3,556 & 1,588 & 1,095 & 2,279 & 4,3 & 15,0 & 1,311 & 1,5 \\
\hline III & 7 & 7,31 & 1,90 & 1,9 & & 0,429 & 17,067 & 8,043 & 3,712 & 4,758 & 2,473 & 1,226 & 0,743 & 3,133 & 4,8 & 16,7 & 1,134 & 2,24 \\
\hline III & 8 & & 3,58 & 2,45 & & & 10,785 & 13,473 & 3,846 & 4,507 & 8,071 & 2,133 & 1,559 & 1,722 & 2,4 & 19,1 & 1,146 & $-0,0$ \\
\hline III & 10 & 11,31 & 4,47 & 2,33 & $0,+1$ & 2,065 & 5,475 & 12,717 & 2,823 & 2,166 & 9,347 & 1,958 & 1,270 & 1,353 & 2,2 & 19,5 & 0,857 & 0,1 \\
\hline III & 12 & 12,46 & 4,23 & 2,47 & 0,34 & 1,394 & 8,941 & 13,930 & 3,142 & 4,309 & 8,395 & 1,837 & 1,523 & 2,972 & 5,0 & 26,1 & 1,134 & 1,22 \\
\hline
\end{tabular}


Tabela 9: Estatística Kolmogorov-Smirnov para as distribuições testadas para as parcelas temporárias nas idades 3 a 6 e 9 a 11 anos, sítio I

Table 9: Kolmogorov-Smirnov statistic for the tested distributions for the temporary sampling plots at ages 3 to 6 and 9 to 11 years - Site I

\begin{tabular}{|c|c|c|c|c|c|c|}
\hline \multirow{2}{*}{ Função } & \multicolumn{6}{|c|}{ Idades } \\
\hline & 3 & 4 & 5 & 6 & 9 & $10-11$ \\
\hline Normal & $0,073^{\text {ns }}$ & $0,091^{\mathrm{ns}}$ & $0,125^{* *}$ & $0,095^{\text {ns }}$ & $0,097^{\mathrm{ns}}$ & $0,076^{\mathrm{ns}}$ \\
\hline Lognormal & $0,126^{\mathrm{ns}}$ & $0,111^{\mathrm{ns}}$ & $0,077^{*}$ & $0,102^{\text {ns }}$ & $0,046^{\mathrm{ns}}$ & $0,142^{\mathrm{ns}}$ \\
\hline Gama & $0,106^{\mathrm{ns}}$ & $0,104^{\mathrm{ns}}$ & $0,094^{* *}$ & $0,091^{\mathrm{ns}}$ & $0,057^{\mathrm{ns}}$ & $0,118^{\mathrm{ns}}$ \\
\hline Weibull 2 & $0,076^{\mathrm{ns}}$ & $0,103^{\mathrm{ns}}$ & $0,152^{* *}$ & $0,089^{\mathrm{ns}}$ & $0,106^{\mathrm{ns}}$ & $0,082^{\mathrm{ns}}$ \\
\hline Weibull 3 & $0,097^{\mathrm{ns}}$ & $0,096^{\mathrm{ns}}$ & $0,095^{\mathrm{ns}}$ & $0,118^{\mathrm{ns}}$ & $0,086^{\mathrm{ns}}$ & $0,120^{\mathrm{ns}}$ \\
\hline Beta & $0,121^{\mathrm{ns}}$ & $0,078^{\mathrm{ns}}$ & $0,105^{* *}$ & $0,078^{\text {ns }}$ & $0,116^{*}$ & $0,135^{\mathrm{ns}}$ \\
\hline $\mathbf{S}_{\mathbf{B}}$ & $0,048^{\mathrm{ns}}$ & $0,048^{\mathrm{ns}}$ & $0,032^{\mathrm{ns}}$ & $0,068^{\mathrm{ns}}$ & $0,051^{\mathrm{ns}}$ & $0,070^{\mathrm{ns}}$ \\
\hline
\end{tabular}

Legenda: ns - não significativo; * - significativo à $5 \%$; ** - significativo à $1 \%$.

Tabela 10: Estatística Kolmogorov-Smirnov para as distribuições testadas para as parcelas temporárias nas idades 3 a 9, 11, 12 e 18 anos no sítio II e para as idades 3 a 10, 12 , 13 e 18 anos no sítio III

Table 10: Kolmogorov-Smirnov statistic for the tested distributions for the temporary sampling plots at ages 3 to 9, 11, 12, and 18 years - Site II, and for the ages 3 to 10, 12 , 13, and 18 years - Site III

\begin{tabular}{|c|c|c|c|c|c|c|c|c|c|c|c|c|c|c|c|c|}
\hline \multirow{3}{*}{ Função } & \multicolumn{8}{|c|}{ Sítio II } & \multicolumn{8}{|c|}{ Sítio III } \\
\hline & \multicolumn{16}{|c|}{ Idades } \\
\hline & 3 & 4 & 5 & 6 & 7 & $8-9$ & 11-12 & 18 & 3 & 4 & 5 & 6 & 7 & $8-9$ & 10 & 12-13 \\
\hline Normal & $0,144^{\mathrm{ns}}$ & $0,106^{\mathrm{ns}}$ & 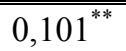 & $0,069^{*}$ & $0,073^{\mathrm{ns}}$ & $0,082^{\mathrm{ns}}$ & $0,081^{*}$ & $0,096^{\mathrm{ns}}$ & $0,149^{\mathrm{ns}}$ & $0,116^{\mathrm{ns}}$ & $0,115^{* *}$ & $0,083^{\mathrm{ns}}$ & $0,111^{* *}$ & $0,082^{\mathrm{ns}}$ & $0,072^{\mathrm{ns}}$ & $0,121^{*}$ \\
\hline Lognormal & $0,127^{\mathrm{ns}}$ & $0,078^{\mathrm{ns}}$ & $0,071^{* *}$ & $0,061^{* *}$ & $0,049^{\mathrm{ns}}$ & $0,042^{\mathrm{ns}}$ & $0,037^{\mathrm{ns}}$ & $0,149^{\mathrm{ns}}$ & $0,159^{\mathrm{ns}}$ & $0,110^{\mathrm{ns}}$ & $0,088^{* *}$ & $0,081^{\mathrm{ns}}$ & $0,073^{* *}$ & $0,136^{\mathrm{ns}}$ & $0,112^{\mathrm{ns}}$ & $0,055^{\mathrm{ns}}$ \\
\hline Gama & $0,135^{\mathrm{ns}}$ & $0,090^{\mathrm{ns}}$ & $0,079^{* *}$ & $0,059^{* *}$ & $0,052^{\mathrm{ns}}$ & $0,056^{\mathrm{ns}}$ & $0,040^{\mathrm{ns}}$ & $0,129^{\mathrm{ns}}$ & $0,161^{\mathrm{ns}}$ & $0,110^{\mathrm{ns}}$ & $0,092^{* *}$ & $0,082^{\mathrm{ns}}$ & $0,082^{* *}$ & $0,116^{\mathrm{ns}}$ & $0,084^{\mathrm{ns}}$ & $0,078^{\mathrm{ns}}$ \\
\hline Weibull 2 & $0,143^{\mathrm{ns}}$ & $0,096^{\mathrm{ns}}$ & $0,138^{* *}$ & $0,088^{* *}$ & $0,080^{* *}$ & $0,081^{\mathrm{ns}}$ & $0,081^{*}$ & $0,107^{\mathrm{ns}}$ & $0,142^{\mathrm{ns}}$ & $0,117^{\mathrm{ns}}$ & $0,158^{* *}$ & $0,097^{\mathrm{ns}}$ & $0,157^{* *}$ & $0,090^{\mathrm{ns}}$ & $0,071^{\mathrm{ns}}$ & $0,113^{\mathrm{ns}}$ \\
\hline Weibull 3 & $0,067^{\mathrm{ns}}$ & $0,072^{\mathrm{ns}}$ & $0,072^{* *}$ & $0,071^{* *}$ & $0,070^{\mathrm{ns}}$ & $0,077^{\mathrm{ns}}$ & $0,068^{\mathrm{ns}}$ & $0,130^{\mathrm{ns}}$ & $0,127^{\mathrm{ns}}$ & $0,129^{\mathrm{ns}}$ & $0,052^{* *}$ & $0,059^{*}$ & $0,056^{* *}$ & $0,152^{\mathrm{ns}}$ & $0,106^{\mathrm{ns}}$ & $0,083^{\mathrm{ns}}$ \\
\hline Beta & $0,090^{\mathrm{ns}}$ & $0,044^{\mathrm{ns}}$ & $0,113^{* *}$ & $0,121^{* *}$ & $0,065^{*}$ & $0,055^{\mathrm{ns}}$ & $0,076^{\mathrm{ns}}$ & $0,130^{\mathrm{ns}}$ & $0,147^{\mathrm{ns}}$ & $0,089^{\mathrm{ns}}$ & $0,117^{* *}$ & $0,089^{\mathrm{ns}}$ & $0,131^{* *}$ & $0,111^{\mathrm{ns}}$ & $0,075^{\mathrm{ns}}$ & $0,080^{\mathrm{ns}}$ \\
\hline $\mathbf{S}_{\mathrm{B}}$ & $0,045^{\mathrm{ns}}$ & $0,022^{\mathrm{ns}}$ & $0,062^{*}$ & $0,055^{*}$ & $0,047^{\mathrm{ns}}$ & $0,026^{\mathrm{ns}}$ & $0,036^{\mathrm{ns}}$ & $0,078^{\mathrm{ns}}$ & $0,151^{\mathrm{ns}}$ & $0,113^{\mathrm{ns}}$ & $0,057^{\mathrm{ns}}$ & $0,069^{\mathrm{ns}}$ & $0,047^{\mathrm{ns}}$ & $0,060^{\mathrm{ns}}$ & $0,034^{\mathrm{ns}}$ & $0,043^{\mathrm{ns}}$ \\
\hline
\end{tabular}

Legenda: ns - não significativo; * - significativo à 5\%; ** - significativo à $1 \%$. 
Tabela 11: Estimativa do número de árvores por classe diamétrica (ha) através da função $\mathrm{S}_{\mathrm{B}}$, por classe diamétrica para as diferentes idades e Sítio III com base nos resultados das parcelas temporárias

Table 11: Number of trees per diameter class (ha) estimated by the $S_{B}$ function for the different ages - Site III, basing on the results of temporary sampling plots

\begin{tabular}{|c|c|c|c|c|c|c|c|c|}
\hline \multirow{2}{*}{$\begin{array}{l}\text { Centro de classe } \\
\text { Diamétrica }(\mathbf{c m})\end{array}$} & \multicolumn{8}{|c|}{ Idades (cm) } \\
\hline & 3 & 4 & 5 & 6 & 7 & $8-9$ & 10 & $12-13$ \\
\hline 1,5 & - & 671,6 & 48,8 & 62,8 & - & - & - & - \\
\hline 2,5 & - & 1835,9 & 163,1 & 233,3 & 0,03 & - & - & - \\
\hline 3,5 & - & 1873,7 & 397,8 & 430,9 & 18,6 & - & 46,0 & - \\
\hline 4,5 & - & 1431,9 & 648,9 & 539,3 & 206,4 & - & 41,0 & - \\
\hline 5,5 & 521,2 & 981,0 & 754,2 & 532,8 & 499,4 & 103,3 & 50,2 & 27,0 \\
\hline 6,5 & 470,9 & 640,1 & 661,9 & 447,1 & 588,7 & 109,1 & 57,2 & 57,5 \\
\hline 7,5 & 370,1 & 407,5 & 461,5 & 330,9 & 480,6 & 154,3 & 62,4 & 87,3 \\
\hline 8,5 & 321,2 & 255,9 & 266,9 & 219,9 & 320,9 & 195,8 & 66,1 & 107,1 \\
\hline 9,5 & 297,6 & 159,2 & 132,7 & 132,1 & 191,0 & 229,8 & 68,6 & 116,4 \\
\hline 10,5 & 290,7 & 98,4 & 58,4 & 71,6 & 106,3 & 253,8 & 69,9 & 117,5 \\
\hline 11,5 & 294,1 & 60,4 & 23,4 & 34,7 & 56,8 & 266,3 & 70,3 & 112,6 \\
\hline 12,5 & 134,2 & 36,8 & 8,6 & 14,8 & 29,7 & 266,8 & 69,7 & 103,9 \\
\hline 13,5 & - & 22,2 & 4,4 & 5,4 & 15,4 & 255,6 & 68,0 & 93,0 \\
\hline 14,5 & - & 13,3 & - & 2,0 & 7,9 & 233,4 & 65,1 & 81,2 \\
\hline 15,5 & - & 18,2 & - & - & 4,1 & 202,0 & 60,9 & 69,3 \\
\hline 16,5 & - & - & - & - & 4,4 & 163,7 & 55,1 & 57,9 \\
\hline 17,5 & - & - & - & - & - & 121,7 & 47,2 & 47,3 \\
\hline 18,5 & - & - & - & - & - & 80,1 & 36,8 & 37,8 \\
\hline 19,5 & - & - & - & - & - & 64,3 & 32,2 & 29,6 \\
\hline 20,5 & - & - & - & - & - & - & - & 22,5 \\
\hline 21,5 & - & - & - & - & - & - & - & 16,6 \\
\hline 22,5 & - & - & - & - & - & - & - & 11,8 \\
\hline 23,5 & - & - & - & - & - & - & - & 8,1 \\
\hline 24,5 & - & - & - & - & - & - & - & 5,2 \\
\hline 25,5 & - & - & - & - & - & - & - & 3,2 \\
\hline 26,5 & - & - & - & - & - & - & - & 3,2 \\
\hline
\end{tabular}

Após a análise, tanto das parcelas permanentes como das temporárias, das quais foram possíveis realizar 372 análises, envolvendo as combinações de sítio, idade e densidade, a função $\mathrm{S}_{\mathrm{B}}$ foi a que melhor se ajustou para a maioria das combinações, apresentando valores não significativos para os testes estatístico $\mathrm{K}-\mathrm{S}$, os quais indicam presença de ajuste, ou seja, adequabilidade do modelo aos dados. Pela análise visual gráfica das curvas ajustadas sobre as os respectivos histogramas e a análise de resíduos extensivamente apresentados em Bartoszeck (2000) a $\mathrm{S}_{\mathrm{B}}$ foi a função que apresentou melhor ajuste e menores resíduos.

Este comportamento foi similar ao trabalho de Finger (1982) com Acacia mearnsii, que, também, comprovou, através dos testes Komogorov-Smirnov e Logarítmo da Probabilidade que a função SB foi a melhor 
para descrever a distribuição de diâmetros em todas as idades estudadas (3,5 a 7,5 anos).

Resultados similares foram encontrados por Thiersch (1997), para Eucalyptus camaldulensis, onde se destacou a função SB de Johnson seguida da função Beta, ambas com ajuste pelo método dos momentos. Observou-se, também, que estas distribuições se mantiveram estáveis para a maioria das idades, muito provavelmente pela grande flexibilidade de suas formas. As outras distribuições embora inferiores à SB, apresentaram desempenhos satisfatórios à exceção das distribuições Gama e Lognormal que apresentaram desempenhos inferiores.

Tabai (1999) estudou a acuracidade da função SB na estimativa da distribuição diamétrica de Pinus taeda por cinco métodos de ajuste para analisar a influência da idade e do espaçamento nos ajustes e avaliar a correlação dos parâmetros estimados pelos vários métodos com a idade, para fazer inferências sobre prognose. Concluiu que o método dos momentos, o mesmo utilizado neste trabalho, deve ser o preferido para o ajuste da equação para esta espécie.

Silva (1999), trabalhando com a produção de Eucalyptus camaldulensis no Estado do Mato Grosso, concluiu para avaliação da estrutura diamétrica que o melhor ajuste foi também conseguido com a função $\mathrm{SB}$ e pelo mesmo método usado no presente trabalho, ou seja, o método dos momentos e a $35 \%$ do diâmetro mínimo, seguidos pelas proporções de 45 e $25 \%$, ocupando as três primeiras posições no ranking.

\section{CONCLUSÕES E RECOMENDAÇÕES}

- A função $S_{B}$ foi a mais eficiente e flexível, propiciando estimativas precisas da distribuição diamétrica de bracatinga na Região Metropolitana de Curitiba, na quase totalidade das combinações de sítio, idade e densidade, demonstrando ser bastante sensível em absorver os efeitos das variações destes fatores. Esta função é indicada para a construção das curvas de distribuição diamétrica.

- Os resultados gerados para as diferentes combinações de sítio, idade e densidade estão em conformidade com o desenvolvimento biológico esperado. Para o fator idade, ocorreu um decréscimo do número de árvores nas classes de DAP inferiores e um aumento do número de árvores nas classes superiores.

- Avaliando o fator sítio, constatou-se que o número de árvores por classe de diâmetro diminui nas classes menores e aumenta nas classes maiores, deslocando a curva da distribuição para a direita, à medida que aumenta os valores de índice de sítio. Ao mesmo tempo, ocorre um achatamento das curvas para o sítio mais produtivo.

- Em relação ao fator densidade, à medida que aumenta a densidade, ocorre um acréscimo do número de árvores nas classes inferiores e um decréscimo nas classes superiores, deslocando a curva da distribuição para a esquerda. $\mathrm{O}$ achatamento vai aumentando da maior para a menor densidade.

Recomenda-se testar outros métodos de ajuste para as distribuições e utilizar as funções de densidade de probabilidade e os atributos do povoamento para realizar uma prognose da distribuição diamétrica do povoamento. Selecionando os atributos mais correlacionados com os parâmetros das funções de distribuição e estimando os mesmos na idade desejada da prognose, permitirá conhecer a nova estrutura diamétrica da floresta até uma idade futura préestabelecida.

A continuidade desta pesquisa deve trazer informações cada vez mais importantes para um melhor aproveitamento da espécie. Recomenda-se ainda, a aplicação de todas estas técnicas para outras espécies florestais nativas como o guanandi e a caxeta, por serem espécies relevantes na economia local das comunidades do litoral e por apresentarem carência de pesquisas voltadas ao manejo mais adequado das mesmas.

\section{REFERÊNCIAS}

BAILEY, R.L.; DELL, T.R. Quantifying diameter distribution with the Weibull function. For. Sci., 19 (2): 97-104. 1973.

BAGGIO, A.J.; CAPARNEZZI, A.A. Biomassa aérea da bracatinga (Mimosa scabrella Benth.) em talhões do sistema de cultivo tradicional. Boletim de Pesquisa Florestal, 34: 31-44, jan./jun. 1997. 
BARROS, P.L.C. Estudo das distribuições diamétricas da floresta do planalto Tapajós Pará. Curitiba, 1980. 123 p. Dissertação (Mestrado em Ciências Florestais) Curso de Engenharia Florestal, Setor de Ciências Agrárias, Universidade Federal do Paraná.

BARTOSZECK, A.C.P.S. Evolução da distribuição diamétrica e da relação hipsométrica em função dos fatores idade, sítio e densidade inicial em bracatingais da região metropolitana de Curitiba. Cu4ritiba, 2000. 214 p. Dissertação (Mestrado em Engenharia Florestal). Curso de Pós-Graduação em Engenharia Florestal, Setor de Ciências Agrárias, Universidade Federal do Paraná.

CLUTTER, J.L.; FORSTON, J.C.; PIENNAR, L.V.; BRISTER, G.H.; BAILEY, R.L. Timber management: a quantitative approach. New York: John Wiley \& Sons, 1983. 333p.

FINGER, C.A.G. Distribuição de diâmetros em acácia negra, Acacia mearnsii de Wild, em diferentes povoamentos e idade. Curitiba, 1982. 129 p. Dissertação (Mestrado em Ciências Florestais), Curso de Pós-Graduação em Engenharia Florestal, Setor de Ciências Agrárias, Universidade Federal do Paraná.

MACHADO, S.A; MARQUESINI, L.C.P.T.; BACOVIS, R. Effects of age, site, average $\mathrm{DBH}$ and density upon the diameter distribution of thinned products from Pinus taeda plantations in the State of Paraná, Brazil. XIX World Congress; In: IUFRO. Montreal, Canadá. 1990.

MACHADO, S.A.; OLIVEIRA, E.B. de; CARPANEZZI, A.A.; BARTOSZECK, A.C.P.S. Classificação de sítio para bracatingais na região metropolitana de Curitiba. Boletim de Pesquisa Florestal. 1997. $n^{\circ} 38$, p. 12-28- EMBRAPA.
SILVA, V.S.M. Produção de Eucalyptus camaldulensis Delnh. no Estado de Mato Grosso. Curitiba, 1999. 179 p. Tese (Doutorado em Ciências Florestais), Curso de Pós-Graduação em Engenharia Florestal, Setor de Ciências Agrárias, Universidade Federal do Paraná.

TABAI, F.C.V. O estudo da acuracidade da distribuição SB para representar a estrutura diamétrica de Pinus taeda, através de cinco métodos de ajuste. Lavras, 1999. 55 p. Dissertação (Mestrado em Ciências Florestais), Curso de Pós-Graduação em Engenharia Florestal, Universidade Federal de Lavras.

THIERSCH, A. Eficiência das distribuições diamétricas para prognose da produção de Eucalyptus camaldulensis. Lavras, 1997. 154 p. Dissertação (Mestrado em Ciências Florestais), Curso de Pós-Graduação em Engenharia Florestal, Universidade Federal de Lavras.

TONON, A.E.N. Efeitos da densidade inicial e do sítio sobre o crescimento e a produção de bracatingais da região metropolitana de Curitiba. Curitiba. Dissertação (Mestrado em Manejo Florestal) - Setor de Ciências Agrárias, Universidade Federal do Paraná. 1998. 209 p.

UMAÑA, C.L.A. Distribuições diamétricas de árvores em dez hectares de floresta tropical úmida da Dois Mil madeireira, Itacoatiara - AM. Manaus: 1997. 44 p. Dissertação (Mestrado em Ciências Florestais), Curso de Pós-Graduação em Biologia Tropical e Recursos Naturais do Convênio Instituto Nacional de Pesquisas da Amazônia, Universidade Federal do Amazonas. 